\title{
Asymptotic analysis for Dirac-harmonic maps from degenerating spin surfaces and with bounded index
}

\author{
Jürgen Jost ${ }^{2} \cdot$ Lei Liu $^{3} \cdot$ Miaomiao $\mathrm{Zhu}^{1}$
}

Received: 12 November 2018 / Accepted: 30 April 2019 / Published online: 24 July 2019

(c) The Author(s) 2019

\begin{abstract}
We study the refined blow-up behaviour of a sequence of Dirac-harmonic maps from degenerating spin surfaces with uniformly bounded energy in the case that the domain surfaces converge to a spin surface with only Neveu-Schwarz type nodes. For Dirac-harmonic necks appearing near the nodes, we show that the limit of the map part of each neck is a geodesic in the target manifold. Moreover, we give a length formula for the limit geodesics appearing near the node in terms of the Pohozaev type constants associated to the sequence. In particular, if the Ricci curvature of the target manifold has a positive lower bound and the Dirac-harmonic sequence has bounded index, then the limit of the map part of the necks consist of geodesics of finite length and the energy identities hold.
\end{abstract}

Mathematics Subject Classification 53C43 - 58E20

\section{Introduction}

Motivated by the nonlinear supersymmetric nonlinear sigma model from quantum field theory, Dirac-harmonic maps are defined as solutions of a harmonic map type system coupled with a Dirac type equation. They were introduced and studied in [2,3]. This subject general-

Communicated by A. Malchiodi.

M. Zhu was supported in part by National Natural Science Foundation of China (No. 11601325).

Miaomiao Zhu

mizhu@sjtu.edu.cn

Jürgen Jost

jost@mis.mpg.de

Lei Liu

leiliu@mis.mpg.de ; lei.liu@math.uni-freiburg.de

1 Present Address: School of Mathematical Sciences, Shanghai Jiao Tong University, 800 Dongchuan Road, Shanghai 200240, People's Republic of China

2 Max Planck Institute for Mathematics in the Sciences, Inselstrasse 22, 04103 Leipzig, Germany

3 Mathematisches Institut, Albert-Ludwigs-Universität Freiburg, Ernst-Zermelo-Strasse 1, 79104

Freiburg im Breisgau, Germany 
izes the theory of harmonic maps and harmonic spinors, which has been widely studied. In dimension two, similarly to harmonic maps, the conformal invariance of the Dirac-harmonic map system leads to non-compactness of the space of Dirac-harmonic maps. In general, bubbling phenomena can occur, due to the possible energy concentration at finitely many points in the domain. When the domain is fixed, the blow-up theory for a sequence of Dirac-harmonic maps with uniformly bounded energy has been systematically studied in $[2,19,26]$ for Diracharmonic maps and in [11] for the case of a more general model. To study the existence problem for Dirac-harmonic maps, a heat flow approach was investigated in $[4,5,12,15]$; see [13-15] for the blow-up analysis of the corresponding approximate Dirac-harmonic maps. Roughly speaking, the results of these works assert that the failure of strong convergence occurs at finitely many energy concentration points. At such points, finitely many bubbles, i.e. non-trivial Dirac-harmonic spheres, can separate, and the sum of the energies of these bubbles accounts for the loss of energy during the process of weak convergence. This is known as the energy identity. Moreover, the image of the map part of the limit Dirac-harmonic map and those of the bubbles are connected in the target manifold. This is called the no neck property.

However, if we allow the domain surface to vary, then the energy identity does not hold in general, see e.g. the example in the case of harmonic maps constructed by Parker [20]. Such a situation was first systematically investigated in [28] where the author proved a generalized energy identity for the sequence when the domain surfaces degenerate to a spin surface with only Neveu-Schwarz type nodes and gave a sufficient and necessary condition that the energy identity holds, extending the case of harmonic maps from degenerating surfaces systematically studied in [29]. In this paper, we shall explore the finer asymptotic behaviour of the Dirac-harmonic necks appearing near the degenerating region of the domain. In the case of harmonic maps, where the spinors are vanishing, such necks converge to geodesics in the target $[7,8,10]$. In the more general case of Dirac-harmonic maps investigated in the present paper, it is natural to conjecture that the limit of the map part of these Dirac-harmonic necks are curves in the target manifold with specific properties similar to geodesics. Also, it should be possible to calculate the lengths of the limiting curves of the necks appearing near the nodes in terms of some geometric quantities associated to the Dirac-harmonic map sequence, as is achieved in the case of harmonic maps in [7,29].

To state our problem more precisely, let $\phi$ be a smooth map from a spin Riemann surface $(M, h)$ with metric $h$ to another compact Riemannian manifold $(N, g)$ with dimension $n \geq 2$. Let $\phi^{\star} T N$ be the pull-back bundle of $T N$ by $\phi$ and consider the twisted bundle $\Sigma M \otimes \phi^{\star} T N$ with induced metric $\langle\cdot, \cdot\rangle_{\Sigma M \otimes \phi^{\star} T N}$ and induced connection $\widetilde{\nabla}$. A smooth section $\psi$ of the twisted bundle $\Sigma M \otimes \phi^{\star} T N$ is called a spinor field along the map $\phi$. Critical points $(\phi, \psi)$ of the action functional

$$
L(\phi, \psi)=\int_{M}\left(|d \phi|^{2}+\langle\psi, \not D \psi\rangle_{\Sigma M \otimes \phi^{\star} T N}\right) d M
$$

are called Dirac-harmonic maps from $M$ to $N$. Here $\not D$ is the Dirac operator along the map $\phi$, defined by $\not D \psi:=e_{\alpha} \cdot \widetilde{\nabla}_{e_{\alpha}} \psi$. See Sect. 2 for more details on the notations and definitions.

Now, let $\left(M_{n}, h_{n}, c_{n}, \sigma_{n}\right)$ be a sequence of closed hyperbolic Riemann surface of genus $g>1$, equipped with hyperbolic metrics $h_{n}$, compatible complex structures $c_{n}$ and spin structures $\sigma_{n}$. We consider a sequence of smooth Dirac-harmonic maps

$$
\left(\phi_{n}, \psi_{n}\right):\left(M_{n}, h_{n}, c_{m}, \sigma_{n}\right) \rightarrow(N, g),
$$

with uniformly bounded energy

$$
E\left(\phi_{n}, \psi_{n} ; M_{n}\right) \leq \Lambda<\infty .
$$


Here the energy of the pair $(\phi, \psi)$ on a domain $\Omega \subset M$ is defined by

$$
E(\phi, \psi ; \Omega)=\int_{\Omega}\left(|\nabla \phi|^{2}+|\psi|^{4}\right) d M .
$$

Denote the energy of $\phi$ on $\Omega \subset M$ by

$$
E(\phi ; \Omega)=\int_{\Omega}|\nabla \phi|^{2} d M
$$

the energy of $\psi$ on $\Omega \subset M$ by

$$
E(\psi ; \Omega)=\int_{\Omega}|\psi|^{4} d M
$$

We shall often omit the domain $M$ from the notation and simply write $E(\phi)=E(\phi ; M)$, $E(\psi)=E(\psi ; M)$ and $E(\phi, \psi)=E(\phi, \psi ; M)$.

In two dimensional geometric variational problems, Pohozaev type identities play an important role in the study of the qualitative behavior for a sequence of solutions. When the domain surface varies and possibly degenerates, one needs to study solutions defined on long cylinders and in such situations, Pohozaev type identities in general no longer hold. Therefore, we need to find some geometric quantities associated to the sequence of solutions and domains to characterize the asymptotic behaviour of the solutions. Such a scheme was first explored in [29] for harmonic maps and then in [28] for Dirac-harmonic maps. Consider a Dirac-harmonic map $(\phi, \psi)$ defined on a standard cylinder $P=\left[t_{1}, t_{2}\right] \times S^{1}$ with flat metric $d s^{2}=d t^{2}+d \theta^{2}$. Denote

$$
T(\phi, \psi)=\left|\phi_{t}\right|^{2}-\left|\phi_{\theta}\right|^{2}+\operatorname{Re}\left\langle\psi, \frac{\partial}{\partial t} \cdot \widetilde{\nabla}_{\frac{\partial}{\partial t}} \psi\right\rangle-2 i\left\langle\phi_{t}, \phi_{\theta}\right\rangle-i \operatorname{Re}\left\langle\psi, \frac{\partial}{\partial t} \cdot \widetilde{\nabla}_{\frac{\partial}{\partial \theta}} \psi\right\rangle .
$$

It is well known that the quadratic differential $T d z^{2}$ is holomorphic. See [3] for details. By Lemma 3.4 in [28] (or Lemma 2.1 in the present paper), we know that

$$
\alpha(\phi, \psi, P):=\int_{\{t\} \times S^{1}} T(\phi, \psi) d \theta
$$

is a complex number which is independent of $t \in\left[t_{1}, t_{2}\right]$. The quantity defined in (1.2) can be considered as a Pohozaev type constant associated to the Dirac-harmonic map $(\phi, \psi)$ defined on the cylinder $P$ and it measures the extent to which the Pohozaev type identity fails. In the case of a vanishing spinor field $\psi \equiv 0$, it reduces to the Pohozaev type constant associated to the harmonic map $\phi$ introduced in [29] (page 64). Such quantities played an important role in the study of the asymptotic behaviour of harmonic maps and Dirac-harmonic maps from degenerating surfaces $[28,29]$. Pohozaev type constants appear also in other two dimensional geometric variational problems, like the singular super-Liouville type systems [16].

Now, we assume that $\left(M_{n}, h_{n}, c_{n}\right)$ degenerates to a hyperbolic Riemann surface $(M, h, c)$ by collapsing $p(1 \leq p \leq 3 g-3)$ pairwise disjoint simple closed geodesics $\gamma_{n}^{j}$ of lengths $l_{n}^{j}$, $j=1, \ldots, p$. For each $j$, the geodesics $\gamma_{n}^{j}$ degenerate into a pair of punctures $\left(\epsilon^{j, 1}, \epsilon^{j, 2}\right)$. Let $P_{n}^{j}$ be the standard cylindrical collar about $\gamma_{n}^{j}$. We associate the sequence $\left(\phi_{n}, \psi_{n}, M_{n}\right)$ with a sequence of $p$-tuples $\left(\alpha_{n}^{1}, \ldots, \alpha_{n}^{p}\right)$, where

$$
\alpha_{n}^{j}:=\alpha\left(\phi_{n}, \psi_{n}, P_{n}^{j}\right) \in \mathbb{C}
$$

are quantities defined by (1.2). Pulling back the hyperbolic metrics $h_{n}$ and the compatible complex structures $c_{n}$ by suitable diffeomorphisms $M \rightarrow M_{n} \backslash \cup_{j=1}^{p} \gamma_{n}^{j}$ and passing to a 
subsequence, we can think of $\left(h_{n}, c_{n}\right)$ as all living on the limit surface $M$, converging in $C_{l o c}^{\infty}$ to $(h, c)$, and the pull back of $\sigma_{n}$ is a fixed spin structure $\sigma$ on $M$. Note that $M$ has $p$ pairs of punctures. As in [28], we require the following assumptions on the limit surface:

All punctures of the limit spin surface $(M, \sigma)$ are of Neveu-Schwarz type.

Then $\sigma$ extends to some spin structure $\bar{\sigma}$ on $\bar{M}$, where $\bar{M}$ is the surface obtained by adding a point at each puncture of $M$. Thus, $\left(\phi_{n}, \psi_{n}\right)$ can be considered as a sequence of Dirac-harmonic maps defined on $\left(M, h_{n}, c_{n}, \sigma\right)$.

In [28], the following generalized energy identities for Dirac-harmonic maps from degenerating surfaces were proved:

Theorem 1.1 Assumptions and notations as above. Then there exist at most finitely many blow-up points $\left\{x_{1}, \ldots, x_{I}\right\}$ which are away from the punctures $\left\{\left(\epsilon^{j, 1}, \epsilon^{j, 2}\right), j=\right.$ $1,2, \ldots, p\}$ and finitely many Dirac-harmonic maps

- $(\phi, \psi):(\bar{M}, \bar{c}, \bar{\sigma}) \rightarrow N$, where $(\bar{M}, \bar{c}, \bar{\sigma})$ is the normalization of $(M, c, \sigma)$,

- $\left(\sigma^{i, l}, \xi^{i, l}\right): S^{2} \rightarrow N, l=1,2, \ldots, L_{i}$, near the blow-up point $x_{i}$,

- $\left(\omega^{j, k}, \zeta^{j, k}\right): S^{2} \rightarrow N, k=1,2, \ldots, K_{j}$, near the $j$-th pair of punctures $\left(\epsilon^{j, 1}, \epsilon^{j, 2}\right)$,

such that after selection of a subsequence, $\left(\phi_{n}, \psi_{n}\right)$ converges to $(\phi, \psi)$ in $C_{l o c}^{\infty} \times C_{l o c}^{\infty}$ on $M \backslash\left\{x_{1}, \ldots, x_{I}\right\}$ and the following identities hold:

$$
\begin{aligned}
& \lim _{n \rightarrow \infty} E\left(\phi_{n}\right)=E(\phi)+\sum_{i=1}^{I} \sum_{l=1}^{L_{i}} E\left(\sigma^{i, l}\right)+\sum_{j=1}^{p} \sum_{k=1}^{K_{j}} E\left(\omega^{j, k}\right)+\sum_{j=1}^{p} \lim _{n \rightarrow \infty}\left|\operatorname{Re} \alpha_{n}^{j}\right| \cdot \frac{2 \pi^{2}}{l_{n}^{j}}, \\
& \lim _{n \rightarrow \infty} E\left(\psi_{n}\right)=E(\psi)+\sum_{i=1}^{I} \sum_{l=1}^{L_{i}} E\left(\xi^{i, l}\right)+\sum_{j=1}^{p} \sum_{k=1}^{K_{j}} E\left(\zeta^{j, k}\right) .
\end{aligned}
$$

When the domain is fixed, it was shown in [19] that the images of the map parts of the weak limit and all the bubbles emerging from any blow-up point are connected, which is known as the no neck property. By slightly modifying the arguments in [19], it is easy to see that the same property holds if the domain surfaces are non-degenerating and stay in a compact region of the moduli space. Therefore, in Theorem 1.1, we know that the image $\phi(M) \cup_{i=1}^{I} \cup_{l=1}^{L_{i}} \sigma^{i, l}$ is a connected set, as the bubbles $\left(\sigma^{i, l}, \xi^{i, l}\right)$ occur at blow-up points that are away from the punctures.

In this paper, we shall explore the finer asymptotic behaviour of the Dirac-harmonic necks that appear near the nodes when the domain surfaces degenerate. It turns out that the map part of these Dirac-harmonic necks converge to geodesics in the target manifold (see Theorem 3.1). To achieve this, we need to carry out a refined neck analysis for Dirac-harmonic maps from long cylinders. Our first main result is the following:

Theorem 1.2 Under the assumptions and notations of Theorem 1.1, the images of $\phi(M)$ and $\omega^{j, k}\left(S^{2}\right), k=1, \ldots, K_{j}, j=1, \ldots, p$ are connected by geodesics on N. Moreover, for each $j=1, \ldots, p$, the asymptotic limit of the sum of the lengths of the geodesics appearing near the $j$-th pair of punctures is

$$
\lim _{n \rightarrow \infty} \sqrt{\left|\operatorname{Re} \alpha_{n}^{j}\right|} \cdot \frac{\sqrt{2 \pi^{3}}}{l_{n}^{j}} .
$$


Remark 1.3 In the above theorem, the length of each geodesic can be finite or infinite and a geodesic of zero length means that the map part of the corresponding neck is converging to a point in the target.

To prove Theorem 1.2, we need to establish a key lemma about the exponential decay along long cylinders of the tangential energies of both the map part and the spinor part. For the case of $\psi_{n} \equiv 0$, i.e. when the $\phi_{n}$ are harmonic maps, this is achieved in [7] where the authors used the ideas in [9] to derive a differential inequality on a long cylinder. However, this kind of technique requires the structure of a harmonic map type equation and cannot be applied to the Dirac equation. Instead, we shall apply the three circle theorem for Dirac-harmonic type systems developed in [19]. The idea is inspired from the work [21], which used a special case of the three circle theorem due to [25] to show that the tangential energy of the sequence on the long cylinder decays exponentially. To derive the exponential decay of the whole energy of spinor, we shall firstly conformally change the long cylinder to an annulus, then we apply a Hardy type inequality as in $[11,15]$ to obtain some differential inequality on this annulus.

According to Theorem 1.1, it is easy to see that the energy identities hold if and only if the following analytical condition is satisfied

$$
\lim _{n \rightarrow \infty}\left|\operatorname{Re} \alpha_{n}^{j}\right| \cdot \frac{2 \pi^{2}}{l_{n}^{j}}=0, \quad j=1, \ldots, p .
$$

A natural question then is whether we can exploit some geometric conditions to ensure that the limiting necks are some geodesics of finite length so that the energy identity follows immediately.

To achieve this, in analogy to the works on minimal hypersurfaces [23] and harmonic maps [18], we shall impose the assumptions that the Ricci curvature of the target manifold has a positive lower bound and the sequence of Dirac-harmonic maps has bounded Morse index (see Sect. 4). In the following, we state our second main result:

Theorem 1.4 Under the assumption of Theorem 1.1, suppose the Ricci curvature of the target manifold $(N, g)$ has a positive lower bound, i.e. there exists a positive constant $\lambda_{0}>0$ such that $\operatorname{Ric}_{N} \geq \lambda_{0}>0$ and assume the sequence $\left(\phi_{n}, \psi_{n}\right)$ has bounded index, then the limit of the map part of the necks consists of geodesics of finite length. Moreover, the energy identities hold, i.e.

$$
\begin{aligned}
& \lim _{n \rightarrow \infty} E\left(\phi_{n}\right)=E(\phi)+\sum_{i=1}^{I} \sum_{l=1}^{L_{i}} E\left(\sigma^{i, l}\right)+\sum_{j=1}^{p} \sum_{k=1}^{K_{j}} E\left(\omega^{j, k}\right), \\
& \lim _{n \rightarrow \infty} E\left(\psi_{n}\right)=E(\psi)+\sum_{i=1}^{I} \sum_{l=1}^{L_{i}} E\left(\xi^{i, l}\right)+\sum_{j=1}^{p} \sum_{k=1}^{K_{j}} E\left(\zeta^{j, k}\right) .
\end{aligned}
$$

We remark that a similar but more subtle and complicated analysis for $\alpha$-Dirac-harmonic maps was carried out in [15], that is, when the functional is perturbed in the sense of [22]. This problem naturally emerges in our context, because our existence scheme for Diracharmonic maps works with such a perturbation to control the asymptotic behavior of our elliptic-parabolic flow.

The rest of the paper is organized as follows. In Sect. 2, we shall review some geometric and analytic aspects of Dirac-harmonic maps. Then we establish the three circle theorem for Dirac-harmonic maps on long cylinders and derive the exponential decay of tangential 
energies of both the map and the spinor. In Sect. 3, we prove our main Theorem 1.2. In Sect. 4, we calculate the second variation formula for the functional $L$, provide the notion of index of Dirac-harmonic maps and then prove Theorem 1.4.

\section{Preliminaries and some basic lemmas}

In this section, we shall first recall the definition of Dirac-harmonic maps and some basic lemmas like the energy gap theorem, the small energy regularity theorem and so on. Then we will establish the three circle lemma for Dirac-harmonic maps on long cylinder which yields the energy exponential decay in the tangential direction.

Let $(M, h, \sigma)$ be a Riemann surface, equipped with a Riemannian metric $h$ and with a fixed spin structure $\sigma, \Sigma M$ be the spinor bundle over $M$ and $\langle\cdot, \cdot\rangle_{\Sigma M}$ be the metric on $\Sigma M$ induced by the Riemannian metric $h$. Choosing a local orthonormal basis $e_{\alpha}, \alpha=1,2$ on $M$, the usual Dirac operator is defined as $\not \partial:=e_{\alpha} \cdot \nabla_{e_{\alpha}}$, where $\nabla$ is the spin connection on $\Sigma M$. The usual Dirac operator $\not \partial$ on a surface can be seen as the Cauchy-Riemann operator. Consider $\mathbb{R}^{2}$ with the Euclidean metric $d x^{2}+d y^{2}$. Let $e_{1}=\frac{\partial}{\partial x}$ and $e_{2}=\frac{\partial}{\partial y}$ be the standard orthonormal frame. A spinor field is simply a map $\psi: \mathbb{R}^{2} \rightarrow \Delta_{2}=\mathbb{C}^{2}$, and the action of $e_{1}$ and $e_{2}$ on spinors can be identified with multiplication with matrices

$$
e_{1}=\left(\begin{array}{cc}
0 & 1 \\
-1 & 0
\end{array}\right), \quad e_{2}=\left(\begin{array}{ll}
0 & i \\
i & 0
\end{array}\right)
$$

If $\psi:=\left(\begin{array}{l}\psi_{1} \\ \psi_{2}\end{array}\right): \mathbb{R}^{2} \rightarrow \mathbb{C}^{2}$ is a spinor field, then the Dirac operator is

$$
\not \partial \psi=\left(\begin{array}{cc}
0 & 1 \\
-1 & 0
\end{array}\right)\left(\begin{array}{c}
\frac{\partial \psi_{1}}{\partial x} \\
\frac{\partial \psi_{2}}{\partial x}
\end{array}\right)+\left(\begin{array}{cc}
0 & i \\
i & 0
\end{array}\right)\left(\begin{array}{c}
\frac{\partial \psi_{1}}{\partial y} \\
\frac{\partial \psi_{2}}{\partial y}
\end{array}\right)=2\left(\begin{array}{c}
\frac{\partial \psi_{2}}{\partial z} \\
-\frac{\partial \psi_{1}}{\partial z}
\end{array}\right),
$$

where

$$
\frac{\partial}{\partial z}=\frac{1}{2}\left(\frac{\partial}{\partial x}-i \frac{\partial}{\partial y}\right), \quad \frac{\partial}{\partial \bar{z}}=\frac{1}{2}\left(\frac{\partial}{\partial x}+i \frac{\partial}{\partial y}\right)
$$

For more details on spin geometry and Dirac operators, one can refer to [17].

Let $\phi$ be a smooth map from $M$ to another compact Riemannian manifold $(N, g)$ with dimension $n \geq 2$. Let $\phi^{\star} T N$ be the pull-back bundle of $T N$ by $\phi$ and then we get the twisted bundle $\Sigma M \otimes \phi^{\star} T N$. Naturally, there is a metric $\langle\cdot, \cdot\rangle_{\Sigma M \otimes \phi^{\star} T N}$ on $\Sigma M \otimes \phi^{\star} T N$ which is induced from the metrics on $\Sigma M$ and $\phi^{\star} T N$. Also we have a natural connection $\widetilde{\nabla}$ on $\Sigma M \otimes \phi^{\star} T N$ which is induced from the connections on $\Sigma M$ and $\phi^{\star} T N$. Let $\psi$ be a section of the bundle $\Sigma M \otimes \phi^{\star} T N$. In local coordinates, it can be written as

$$
\psi=\psi^{i} \otimes \partial_{y^{i}}(\phi),
$$

where each $\psi^{i}$ is a usual spinor on $M$ and $\partial_{y^{i}}$ is the nature local basis on $N$. Then $\widetilde{\nabla}$ becomes

$$
\widetilde{\nabla} \psi=\nabla \psi^{i} \otimes \partial_{y^{i}}(\phi)+\left(\Gamma_{j k}^{i} \nabla \phi^{j}\right) \psi^{k} \otimes \partial_{y^{i}}(\phi)
$$

where $\Gamma_{j k}^{i}$ are the Christoffel symbols of the Levi-Civita connection of $N$. The Dirac operator along the map $\phi$ is defined by $\not D \psi:=e_{\alpha} \cdot \widetilde{\nabla}_{e_{\alpha}} \psi$. 
We consider the following functional

$$
\begin{aligned}
L(\phi, \psi) & =\int_{M}\left(|d \phi|^{2}+\langle\psi, \not D \psi\rangle_{\Sigma M \otimes \phi^{\star} T N}\right) d M \\
& =\int_{M}\left(g_{i j}(\phi) h^{\alpha \beta} \frac{\partial \phi^{i}}{\partial x^{\alpha}} \frac{\partial \phi^{j}}{\partial x^{\beta}}+g_{i j}(\phi)\left\langle\psi^{i}, \not D \psi^{j}\right\rangle_{\Sigma M}\right) d M .
\end{aligned}
$$

The functional $L(\phi, \psi)$ is conformally invariant. That is , for any conformal diffeomorphism $f: M \rightarrow M$, setting

$$
\widetilde{\phi}=\phi \circ f \quad \text { and } \quad \widetilde{\psi}=\lambda^{-1 / 2} \psi \circ f .
$$

Then $L(\widetilde{\phi}, \widetilde{\psi})=L(\phi, \psi)$. Here $\lambda$ is the conformal factor of the conformal map $f$, i.e. $f^{*} h=\lambda^{2} h$. Critical points $(\phi, \psi)$ of the action functional $L$ are called Dirac-harmonic maps from $M$ to $N$.

The Euler-Lagrange equations of the functional $L$ are

$$
\begin{gathered}
\left(\Delta \phi^{i}+\Gamma_{j k}^{i} h^{\alpha \beta} \phi_{\alpha}^{j} \phi_{\beta}^{k}\right) \frac{\partial}{\partial y^{i}}(\phi(x))=R(\phi, \psi), \\
\not D \psi=0,
\end{gathered}
$$

where $R(\phi, \psi)$ is defined by

$$
R(\phi, \psi)=\frac{1}{2} R_{l i j}^{m}(\phi(x))\left\langle\psi^{i}, \nabla \phi^{l} \cdot \psi^{j}\right\rangle \frac{\partial}{\partial y^{m}}(\phi(x)) .
$$

Here $R_{l i j}^{m}$ stands for the Riemann curvature tensor of the target manifold $(N, g)$.

By the Nash embedding theorem, we embed $N$ into $\mathbb{R}^{K}$. Then, the critical points $(\phi, \psi)$ satisfy the Euler-Lagrange equations

$$
\Delta \phi=A(\phi)(d \phi, d \phi)+\operatorname{Re}\left(P\left(\mathcal{A}\left(d \phi\left(e_{\alpha}\right), e_{\alpha} \cdot \psi\right) ; \psi\right)\right),
$$

$$
\not \partial \psi=\mathcal{A}\left(d \phi\left(e_{\alpha}\right), e_{\alpha} \cdot \psi\right),
$$

where $\not \partial$ is the usual Dirac operator, $A$ is the second fundamental form of $N$ in $\mathbb{R}^{K}$, and

$$
\begin{aligned}
\mathcal{A}\left(d \phi\left(e_{\alpha}\right), e_{\alpha} \cdot \psi\right) & :=\left(\nabla \phi^{i} \cdot \psi^{j}\right) \otimes A\left(\partial_{y^{i}}, \partial_{y^{j}}\right), \\
\operatorname{Re}\left(P\left(\mathcal{A}\left(d \phi\left(e_{\alpha}\right), e_{\alpha} \cdot \psi\right) ; \psi\right)\right) & :=P\left(A\left(\partial_{y^{l}}, \partial_{y^{j}}\right) ; \partial_{y^{i}}\right) \operatorname{Re}\left(\left\langle\psi^{i}, d \phi^{l} \cdot \psi^{j}\right\rangle\right) .
\end{aligned}
$$

Here $P(\xi ; \cdot)$ denotes the shape operator, defined by $\langle P(\xi ; X), Y\rangle=\langle A(X, Y), \xi\rangle$ for $X, Y \in$ $\Gamma(T N)$, and $\operatorname{Re}(z)$ denotes the real part of $z \in \mathbb{C}$. We refer to [2,6,11,24,27] for more details.

Next, we recall some basic lemmas which will be used in this paper.

Lemma 2.1 (Lemma 3.4 in [28]). Let $(\phi, \psi):\left[T_{1}, T_{2}\right] \times S^{1} \rightarrow N$ be a Dirac-harmonic map. Then

$$
\alpha(\phi, \psi):=\int_{\{t\} \times S^{1}} T(\phi, \psi) d \theta
$$

is independent of $t \in\left[T_{1}, T_{2}\right]$, where $T(\phi, \psi)$ is defined in (1.1). 
Lemma 2.2 (Theorem 3.1 in [2]). Let $M$ be a closed spin Riemann surface with a fixed spin structure and $N$ be a compact Riemannian manifold. Then there is a small constant $\epsilon_{0}>0$ such that if $(\phi, \psi): M \rightarrow N$ is a smooth Dirac-harmonic map satisfying

$$
\int_{M}\left(|d \phi|^{2}+|\psi|^{4}\right) d M \leq \epsilon_{0},
$$

then $\phi$ is a constant map and $\psi$ is a tuple of harmonic spinors.

Lemma 2.3 (Theorem 3.2 in [2]). There is a small constant $\epsilon_{0}>0$ such that if $(\phi, \psi): D \rightarrow$ $N$ is a smooth Dirac-harmonic map where $D \subset \mathbb{R}^{2}$ is the unit disk, satisfying

$$
\int_{D}\left(|d \phi|^{2}+|\psi|^{4}\right) d M \leq \epsilon_{0}
$$

then

$$
\begin{aligned}
& \|d \phi\|_{W^{1, p}\left(D_{\frac{1}{2}}\right)} \leq C(p, N)\|d \phi\|_{L^{2}(D)},\|\nabla \psi\|_{W^{1, p}\left(D_{\frac{1}{2}}\right)} \leq C(p, N)\|\psi\|_{L^{4}(D)}, \\
& \|d \phi\|_{L^{\infty}\left(D_{\frac{1}{2}}\right)} \leq C(N)\|d \phi\|_{L^{2}(D)},\|\psi\|_{L^{\infty}\left(D_{\frac{1}{2}}\right)} \leq C(N)\|\psi\|_{L^{4}(D)} .
\end{aligned}
$$

Lemma 2.4 (Theorem 4.6 in [3]). Let $(\phi, \psi): D \backslash\{0\} \rightarrow N$ be a smooth Dirac-harmonic map with finite energy $E(\phi, \psi ; D) \leq \Lambda<\infty$, then $(\phi, \psi)$ can be extended smoothly to the whole disk $D$.

Let $L>0$ be a constant, denote $P_{i}=[(i-1) L, i L] \times S^{1}$ and

$$
\|(u, v)\|_{L^{2}\left(P_{i}\right)}^{2}=\int_{P_{i}}\left(|u|^{2}+|v|^{2}\right) d t d \theta .
$$

Proposition 2.5 (Proposition 3.3 in [19]). Suppose $(u, v) \in C^{\infty}\left(P_{i-1} \cup P_{i} \cup P_{i+1}\right)$ satisfy equations

$$
\begin{aligned}
& \Delta u=A^{1} u+A^{2} \nabla u+A^{3} v+\frac{1}{2 \pi} \int_{0}^{2 \pi} A^{4} u+A^{5} \nabla u+A^{6} v d \theta, \\
& \not \partial v=B^{1} u+B^{2} \nabla u+B^{3} v+\frac{1}{2 \pi} \int_{0}^{2 \pi} B^{4} u+B^{5} \nabla u+B^{6} v d \theta,
\end{aligned}
$$

with $L$ is given and large. Then there exists a positive constant $\delta_{0}$ such that if $\left\|A^{j}\right\|_{L^{\infty}} \leq \delta_{0}$, $\left\|B^{j}\right\|_{L^{\infty}} \leq \delta_{0}$ for $j=1, \ldots, 6$ and

$$
\int_{(i-1) L \times S^{1}} u d \theta, \int_{(i-1) L \times S^{1}} v d \theta, \int_{i L \times S^{1}} u d \theta, \int_{i L \times S^{1}} v d \theta \leq \delta_{0},
$$


then

(a) $\|(u, v)\|_{L^{2}\left(P_{i+1}\right)} \leq e^{-\frac{1}{2} L}\|(u, v)\|_{L^{2}\left(P_{i}\right)}$ implies $\|(u, v)\|_{L^{2}\left(P_{i}\right)} \leq e^{-\frac{1}{2} L}\|(u, v)\|_{L^{2}\left(P_{i-1}\right)}$;

(b) $\|(u, v)\|_{L^{2}\left(P_{i-1}\right)} \leq e^{-\frac{1}{2} L}\|(u, v)\|_{L^{2}\left(P_{i}\right)}$ implies $\|(u, v)\|_{L^{2}\left(P_{i}\right)} \leq e^{-\frac{1}{2} L}\|(u, v)\|_{L^{2}\left(P_{i+1}\right)}$;

(c) either $\|(u, v)\|_{L^{2}\left(P_{i}\right)} \leq e^{-\frac{1}{2} L}\|(u, v)\|_{L^{2}\left(P_{i+1}\right)}$ or $\|(u, v)\|_{L^{2}\left(P_{i}\right)} \leq e^{-\frac{1}{2} L}\|(u, v)\|_{L^{2}\left(P_{i-1}\right)}$.

Lemma 2.6 (Lemma 3.1 in [19]). Suppose $(u, v) \in C^{\infty}\left([-2,2] \times S^{1}\right)$ satisfy (2.7)-(2.8). Assume $A^{j}, B^{j} \in C^{\infty}\left([-2,2] \times S^{1}\right), j=1, \ldots, 6$ and $\sum_{j=1}^{6}\left\|A^{j}\right\|_{L^{\infty}}+\sum_{j=1}^{6}\left\|B^{j}\right\|_{L^{\infty}} \leq$ $C<\infty$, then

$$
\begin{aligned}
\|u\|_{W^{2,2}\left([-1,1] \times S^{1}\right)} & \leq C\left(\|u\|_{L^{2}\left([-2,2] \times S^{1}\right)}+\|v\|_{L^{2}\left([-2,2] \times S^{1}\right)}\right), \\
\|v\|_{W^{1,2}\left([-1,1] \times S^{1}\right)} & \leq C\left(\|u\|_{L^{2}\left([-2,2] \times S^{1}\right)}+\|v\|_{L^{2}\left([-2,2] \times S^{1}\right)}\right) .
\end{aligned}
$$

In the end of this section, we derive the exponential decay of the tangential energy on the long cylinder. Set

$$
Q_{t}(k):=[t-k, t+k] \times S^{1} .
$$

Lemma 2.7 Let $N$ be a compact Riemannian manifold. Let $\left(\phi_{n}, \psi_{n}\right):\left[-T_{n}, T_{n}\right] \times S^{1} \rightarrow N$ be a sequence of Dirac-harmonic maps with uniformly bounded energy

$$
E\left(\phi_{n}, \psi_{n} ;\left[-T_{n}, T_{n}\right] \times S^{1}\right) \leq \Lambda,
$$

where $\left[-T_{n}, T_{n}\right] \times S^{1}$ is a cylinder with standard flat metric $d s^{2}=d t^{2}+d \theta^{2}$ and $T_{n} \rightarrow \infty$ as $n \rightarrow \infty$.

If there is no energy concentration for $\left(\phi_{n}, \psi_{n}\right)$, then for any fixed $k>0$ and for any $t_{n} \in\left[-T_{n}+T+k, T_{n}-T-k\right]$, when $n$ and $T$ are sufficiently large, there holds

$$
\begin{aligned}
& \left(\int_{Q_{t_{n}}(k)}\left|\frac{\partial \phi_{n}}{\partial \theta}\right|^{2} d t d \theta\right)^{\frac{1}{2}}+\left(\int_{Q_{t_{n}}(k)}\left|\frac{\partial \psi_{n}}{\partial \theta}\right|^{2} d t d \theta\right)^{\frac{1}{2}}+\left(\int_{Q_{t_{n}}(k)}\left|\frac{\partial \psi_{n}}{\partial \theta}\right|^{\frac{4}{3}} d t d \theta\right)^{\frac{3}{4}} \\
& \leq C\left(e^{-\frac{t_{n}-k-\left(-T_{n}+T\right)}{2}}+e^{-\frac{T_{n}-T-\left(t_{n}+k\right)}{2}}\right) o(n, T),
\end{aligned}
$$

where $\lim _{T \rightarrow \infty} \lim _{n \rightarrow \infty} o(n, T)=0$ and $C=C(L)$ is a positive constant, $L$ is the positive constant in Proposition 2.5.

Proof The idea is similar to the argument in the proof of Theorem 1.4 in [19]. Since there is no energy concentration for $\left(\phi_{n}, \psi_{n}\right)$, by a standard contradiction argument in $[10,26]$, we have

$$
\lim _{T \rightarrow \infty} \lim _{n \rightarrow \infty} \sup _{t \in\left[-T_{n}+T+2, T_{n}-T-2\right]} E\left(\phi_{n}, \psi_{n} ;[t-2, t+2] \times S^{1}\right)=0 .
$$

This means that for any $\epsilon>0$, there exist two positive integers $T_{\epsilon}$ and $N_{\epsilon}$ which are large enough such that when $n \geq N_{\epsilon}$, there holds

$$
\sup _{t \in\left[-T_{n}+T_{\epsilon}+2, T_{n}-T_{\epsilon}-2\right]} E\left(\phi_{n}, \psi_{n} ;[t-2, t+2] \times S^{1}\right) \leq \epsilon .
$$

Now, by (2.10) and Lemma 2.3, we have that for any small $\epsilon>0$, when $n$ and $T$ are large enough, there holds

$$
\left\|d \phi_{n}\right\|_{L^{\infty}\left(\left[-T_{n}+T, T_{n}-T\right] \times S^{1}\right)}+\left\|\psi_{n}\right\|_{L^{\infty}\left(\left[-T_{n}+T, T_{n}-T\right] \times S^{1}\right)} \leq \epsilon .
$$


Denote

$$
\phi_{n}^{*}(t)=\frac{1}{2 \pi} \int_{0}^{2 \pi} \phi_{n}(t, \theta) d \theta, \quad \psi_{n}^{*}(t)=\frac{1}{2 \pi} \int_{0}^{2 \pi} \psi_{n}(t, \theta) d \theta,
$$

and

$$
u=\phi_{n}-\phi_{n}^{*}, v=\psi_{n}-\psi_{n}^{*},
$$

by a direct computation as in [19], we can prove that $(u, v)$ satisfy Eqs. (2.7)-(2.8) (see equations (3.17) and (3.18) in [19]). Moreover, using the estimate (2.11), $(u, v)$ satisfy the conditions in Proposition 2.5 (see also [19] for details).

For simplicity of notations, we also set

$$
P_{i}=\left[-T_{n}+T+(i-1) L,-T_{n}+T+i L\right] \times S^{1},
$$

where $L>0$ is a constant in Proposition 2.5. Without loss of generality, we may assume

$$
2\left(T_{n}-T\right)=k_{n} L,
$$

where $k_{n}$ is a positive integer which goes to infinity as $n \rightarrow \infty$.

By $(c)$ of Proposition 2.5, we obtain

$$
\|(u, v)\|_{L^{2}\left(P_{i}\right)} \leq e^{-\frac{1}{2} L}\|(u, v)\|_{L^{2}\left(P_{i+1}\right)} \text { or }\|(u, v)\|_{L^{2}\left(P_{i}\right)} \leq e^{-\frac{1}{2} L}\|(u, v)\|_{L^{2}\left(P_{i-1}\right)} .
$$

Then, using the $(a)$ and $(b)$ of Proposition 2.5, by iterating, we have

$$
\|(u, v)\|_{L^{2}\left(P_{i}\right)} \leq e^{-\frac{i}{2} L}\|(u, v)\|_{L^{2}\left(P_{1}\right)} \text { or }\|(u, v)\|_{L^{2}\left(P_{i}\right)} \leq e^{-\frac{k_{n}-i}{2} L}\|(u, v)\|_{L^{2}\left(P_{k_{n}}\right)} .
$$

This yields

$$
\begin{aligned}
\left\|\phi_{n}-\phi_{n}^{*}\right\|_{L^{2}\left(P_{i}\right)} \leq\left(e^{-\frac{i}{2} L}+e^{-\frac{k_{n}-i}{2} L}\right)\left(\left\|\phi_{n}-\phi_{n}^{*}\right\|_{L^{2}\left(P_{1}\right)}+\left\|\phi_{n}-\phi_{n}^{*}\right\|_{L^{2}\left(P_{k_{n}}\right)}\right), \\
\left\|\psi_{n}-\psi_{n}^{*}\right\|_{L^{2}\left(P_{i}\right)} \leq\left(e^{-\frac{i}{2} L}+e^{-\frac{k_{n}-i}{2} L}\right)\left(\left\|\phi_{n}-\phi_{n}^{*}\right\|_{L^{2}\left(P_{1}\right)}+\left\|\phi_{n}-\phi_{n}^{*}\right\|_{L^{2}\left(P_{k_{n}}\right)}\right) .
\end{aligned}
$$

Applying the interior estimates Lemma 2.6, we get

$$
\begin{aligned}
\left\|\frac{\partial \phi_{n}}{\partial \theta}\right\|_{L^{2}\left(P_{i}\right)} & \leq\left\|\nabla\left(\phi_{n}-\phi_{n}^{*}\right)\right\|_{L^{2}\left(P_{i}\right)} \\
& \leq C\left(\left\|\phi_{n}-\phi_{n}^{*}\right\|_{L^{2}\left(P_{i} \cup P_{i-1} \cup P_{i+1}\right)}+\left\|\psi_{n}-\psi_{n}^{*}\right\|_{L^{2}\left(P_{i} \cup P_{i-1} \cup P_{i+1}\right)}\right) \\
& \leq C\left(e^{-\frac{i}{2} L}+e^{-\frac{k_{n}-i}{2} L}\right)\left(\left\|\phi_{n}-\phi_{n}^{*}\right\|_{L^{2}\left(P_{1} \cup P_{k_{n}}\right)}+\left\|\psi_{n}-\psi_{n}^{*}\right\|_{L^{2}\left(P_{1} \cup P_{k_{n}}\right)}\right)
\end{aligned}
$$

and

$$
\begin{aligned}
\left\|\frac{\partial \psi_{n}}{\partial \theta}\right\|_{L^{2}\left(P_{i}\right)} & \leq\left\|\nabla\left(\psi_{n}-\psi_{n}^{*}\right)\right\|_{L^{2}\left(P_{i}\right)} \\
& \leq C\left(\left\|\phi_{n}-\phi_{n}^{*}\right\|_{L^{2}\left(P_{i} \cup P_{i-1} \cup P_{i+1}\right)}+\left\|\psi_{n}-\psi_{n}^{*}\right\|_{L^{2}\left(P_{i} \cup P_{i-1} \cup P_{i+1}\right)}\right) \\
& \leq C\left(e^{-\frac{i}{2} L}+e^{-\frac{k_{n}-i}{2} L}\right)\left(\left\|\phi_{n}-\phi_{n}^{*}\right\|_{L^{2}\left(P_{1} \cup P_{k_{n}}\right)}+\left\|\psi_{n}-\psi_{n}^{*}\right\|_{L^{2}\left(P_{1} \cup P_{k_{n}}\right)}\right) .
\end{aligned}
$$


Then it is easy to see that the conclusion of the lemma follows immediately from the above decay estimates. In fact, there exist two positive integers $i_{1}, i_{2}$, such that $1 \leq i_{1} \leq i_{2} \leq k_{n}$ and

$$
\begin{aligned}
& -T_{n}+T+\left(i_{1}-1\right) L \leq t_{n}-k<-T_{n}+T+i_{1} L, \\
& -T_{n}+T+\left(i_{2}-1\right) L \leq t_{n}+k<-T_{n}+T+i_{2} L .
\end{aligned}
$$

According to (2.14) and (2.15), we have

$$
\begin{aligned}
& \left\|\frac{\partial \phi_{n}}{\partial \theta}\right\|_{L^{2}\left(Q_{t_{n}}(k)\right)}+\left\|\frac{\partial \psi_{n}}{\partial \theta}\right\|_{L^{2}\left(Q_{t_{n}}(k)\right)}+\left\|\frac{\partial \psi_{n}}{\partial \theta}\right\|_{L^{\frac{4}{3}}\left(Q_{t_{n}}(k)\right)} \\
& \leq \sum_{i=i_{1}}^{i_{2}}\left(\left\|\frac{\partial \phi_{n}}{\partial \theta}\right\|_{L^{2}\left(P_{i}\right)}+\left\|\frac{\partial \psi_{n}}{\partial \theta}\right\|_{L^{2}\left(P_{i}\right)}+\left\|\frac{\partial \psi_{n}}{\partial \theta}\right\|_{L^{\frac{4}{3}\left(P_{i}\right)}}\right) \\
& \leq C(L) \sum_{i=i_{1}}^{i_{2}}\left(\left\|\frac{\partial \phi_{n}}{\partial \theta}\right\|_{L^{2}\left(P_{i}\right)}+\left\|\frac{\partial \psi_{n}}{\partial \theta}\right\|_{L^{2}\left(P_{i}\right)}\right) \\
& \leq C \sum_{i=i_{1}}^{i_{2}}\left(e^{-\frac{i}{2} L}+e^{-\frac{k_{n}-i}{2} L}\right)\left(\left\|\phi_{n}-\phi_{n}^{*}\right\|_{L^{2}\left(P_{1} \cup P_{k_{n}}\right)}+\left\|\psi_{n}-\psi_{n}^{*}\right\|_{L^{2}\left(P_{1} \cup P_{k_{n}}\right)}\right) \\
& \leq C\left(e^{-\frac{i_{1} L}{2}}+e^{-\frac{\left(k_{n}-i_{2}\right) L}{2}}\right)\left(\left\|\phi_{n}-\phi_{n}^{*}\right\|_{L^{2}\left(P_{1} \cup P_{k_{n}}\right)}+\left\|\psi_{n}-\psi_{n}^{*}\right\|_{L^{2}\left(P_{1} \cup P_{k_{n}}\right)}\right) \\
& \leq C\left(e^{-\frac{t_{n}-k-\left(-T_{n}+T\right)}{2}}+e^{-\frac{T_{n}-T-\left(t_{n}+k\right)}{2}}\right)\left(\left\|\phi_{n}-\phi_{n}^{*}\right\|_{L^{2}\left(P_{1} \cup P_{k_{n}}\right)}+\left\|\psi_{n}-\psi_{n}^{*}\right\|_{L^{2}\left(P_{1} \cup P_{k_{n}}\right)}\right) .
\end{aligned}
$$

Lastly, by (2.11), it is easy to see that

$$
\left(\left\|\phi_{n}-\phi_{n}^{*}\right\|_{L^{2}\left(P_{1} \cup P_{k_{n}}\right)}+\left\|\psi_{n}-\psi_{n}^{*}\right\|_{L^{2}\left(P_{1} \cup P_{k_{n}}\right)}\right)=o(n, T) .
$$

We finished the proof of the lemma.

As a corollary of the above lemma, we have

Lemma 2.8 Let $N$ be a compact Riemannian manifold. Let $\left(\phi_{n}, \psi_{n}\right):\left[-T_{n}, T_{n}\right] \times S^{1} \rightarrow N$ be a sequence of Dirac-harmonic maps with uniformly bounded energy

$$
E\left(\phi_{n}, \psi_{n} ;\left[-T_{n}, T_{n}\right] \times S^{1}\right) \leq \Lambda,
$$

where $\left[-T_{n}, T_{n}\right] \times S^{1}$ is a cylinder with standard flat metric $d s^{2}=d t^{2}+d \theta^{2}$ and $T_{n} \rightarrow \infty$ as $n \rightarrow \infty$.

Suppose there is no energy concentration for $\left(\phi_{n}, \psi_{n}\right)$, then there hold

(1) if $\lim _{n \rightarrow \infty}\left|\operatorname{Re} \alpha\left(\phi_{n}, \psi_{n}\right)\right| T_{n}=0$, then

$$
\lim _{T \rightarrow \infty} \lim _{n \rightarrow \infty} E\left(\phi_{n}, \psi_{n} ;\left[-T_{n}+T, T_{n}-T\right] \times S^{1}\right)=0 ;
$$

(2) if $\lim _{n \rightarrow \infty} \sqrt{\left|\operatorname{Re} \alpha\left(\phi_{n}, \psi_{n}\right)\right|} T_{n}=0$, then

$$
\lim _{T \rightarrow \infty} \lim _{n \rightarrow \infty} \operatorname{Osc}_{\left[-T_{n}+T, T_{n}-T\right] \times S^{1}} \phi_{n}=0 .
$$

Proof Noting that

$$
\left|\widetilde{\nabla}_{\frac{\partial}{\partial \theta}} \psi_{n}\right| \leq C(N)\left(\left|\frac{\partial \psi_{n}}{\partial \theta}\right|+\left|\frac{\partial \phi_{n}}{\partial \theta}\right|\left|\psi_{n}\right|\right)
$$


and

$$
\not D \psi_{n}=\frac{\partial}{\partial t} \cdot \widetilde{\nabla}_{\frac{\partial}{\partial t}} \psi_{n}+\frac{\partial}{\partial \theta} \cdot \widetilde{\nabla}_{\frac{\partial}{\partial \theta}} \psi_{n}=0
$$

by Lemma 2.1, we have

$$
\begin{aligned}
& \int_{\left[-T_{n}+T, T_{n}-T\right] \times S^{1}}\left|\nabla \phi_{n}\right|^{2} d t d \theta \\
& =\int_{\left[-T_{n}+T, T_{n}-T\right] \times S^{1}}\left|\frac{\partial \phi_{n}}{\partial \theta}\right|^{2} d t d \theta+\int_{\left[-T_{n}+T, T_{n}-T\right] \times S^{1}}\left|\frac{\partial \phi_{n}}{\partial t}\right|^{2} d t d \theta \\
& =2 \int_{\left[-T_{n}+T, T_{n}-T\right] \times S^{1}}\left|\frac{\partial \phi_{n}}{\partial \theta}\right|^{2} d t d \theta+\int_{\left[-T_{n}+T, T_{n}-T\right] \times S^{1}} \operatorname{Re}\left\langle\psi_{n}, \frac{\partial}{\partial \theta} \cdot \widetilde{\nabla}_{\frac{\partial}{\partial \theta}} \psi_{n}\right\rangle \\
& +2\left(T_{n}-T\right) \operatorname{Re} \alpha\left(\phi_{n}, \psi_{n}\right) \\
& \leq 2 \int_{\left[-T_{n}+T, T_{n}-T\right] \times S^{1}}\left|\frac{\partial \phi_{n}}{\partial \theta}\right|^{2} d t d \theta+2\left(T_{n}-T\right)\left|\operatorname{Re} \alpha\left(\phi_{n}, \psi_{n}\right)\right| \\
& +C(N) \int_{\left[-T_{n}+T, T_{n}-T\right] \times S^{1}}\left(\left|\psi_{n}\right|^{2}\left|\frac{\partial \phi_{n}}{\partial \theta}\right|+\left|\psi_{n}\right|\left|\frac{\partial \psi_{n}}{\partial \theta}\right|\right) \\
& \leq 2 \int_{\left[-T_{n}+T, T_{n}-T\right] \times S^{1}}\left|\frac{\partial \phi_{n}}{\partial \theta}\right|^{2} d t d \theta+2\left(T_{n}-T\right)\left|\operatorname{Re} \alpha\left(\phi_{n}, \psi_{n}\right)\right| \\
& +C(N)\left(\int_{\left[-T_{n}+T, T_{n}-T\right] \times S^{1}}\left|\psi_{n}\right|^{4}\right)^{\frac{1}{2}}\left(\int_{\left[-T_{n}+T, T_{n}-T\right] \times S^{1}}\left|\frac{\partial \phi_{n}}{\partial \theta}\right|^{2}\right)^{\frac{1}{2}} \\
& +C(N)\left(\int_{\left[-T_{n}+T, T_{n}-T\right] \times S^{1}}\left|\psi_{n}\right|^{4}\right)^{\frac{1}{4}}\left(\int_{\left[-T_{n}+T, T_{n}-T\right] \times S^{1}}\left|\frac{\partial \psi_{n}}{\partial \theta}\right|^{\frac{4}{3}}\right)^{\frac{3}{4}} .
\end{aligned}
$$

We recall the notation

$$
P_{i}=\left[-T_{n}+T+(i-1) L,-T_{n}+T+i L\right] \times S^{1},
$$

and without loss of generality, we assume $2\left(T_{n}-T\right)=k_{n} L$ where $L$ is the positive constant in Proposition 2.5 and $k_{n}$ is a positive integer which will go to infinity as $n \rightarrow \infty$.

By Lemma 2.7, we get

$$
\begin{aligned}
\int_{\left[-T_{n}+T, T_{n}-T\right] \times S^{1}}\left|\frac{\partial \phi_{n}}{\partial \theta}\right|^{2} & =\sum_{i=1}^{k_{n}} \int_{P_{i}}\left|\frac{\partial \phi_{n}}{\partial \theta}\right|^{2} \\
& \leq C \sum_{i=1}^{k_{n}}\left(e^{-\frac{-T_{n}+T+(i-1) L-\left(-T_{n}+T\right)}{2}}+e^{-\frac{T_{n}-T-\left(-T_{n}+T+i L\right)}{2}}\right) o(n, T) \\
& =C \sum_{i=1}^{k_{n}}\left(e^{-\frac{(i-1) L}{2}}+e^{-\frac{2\left(T_{n}-T\right)-i L}{2}}\right) o(n, T) \\
& \leq C \sum_{i=1}^{k_{n}}\left(e^{-\frac{i}{2} L}+e^{-\frac{k_{n}-i}{2} L}\right) o(n, T) \leq \operatorname{Co}(n, T)
\end{aligned}
$$


Similarly,

$$
\begin{aligned}
\int_{\left[-T_{n}+T, T_{n}-T\right] \times S^{1}}\left|\frac{\partial \psi_{n}}{\partial \theta}\right|^{\frac{4}{3}} & =\sum_{i=1}^{k_{n}} \int_{P_{i}}\left|\frac{\partial \psi_{n}}{\partial \theta}\right|^{\frac{4}{3}} \\
& \leq C(L) \sum_{i=1}^{k_{n}} \int_{P_{i}}\left|\frac{\partial \psi_{n}}{\partial \theta}\right|^{2} \\
& \leq C \sum_{i=1}^{k_{n}}\left(e^{-\frac{i}{2} L}+e^{-\frac{k_{n}-i}{2} L}\right) o(n, T) \leq C o(n, T) .
\end{aligned}
$$

Combining (2.16) with (2.17) and (2.18), we obtain

$$
\lim _{T \rightarrow \infty} \lim _{n \rightarrow \infty} \int_{\left[-T_{n}+T, T_{n}-T\right] \times S^{1}}\left|\nabla \phi_{n}\right|^{2} d t d \theta=0 .
$$

In order to prove

$$
\lim _{T \rightarrow \infty} \lim _{n \rightarrow \infty} \int_{\left[-T_{n}+T, T_{n}-T\right] \times S^{1}}\left|\psi_{n}\right|^{4} d t d \theta=0,
$$

taking a cut-off function $\eta \in C_{0}^{\infty}\left(\left(-T_{n}+T, T_{n}-T\right) \times S^{1}\right)$ such that

$$
0 \leq \eta \leq 1,\left.\eta\right|_{\left(-T_{n}+T+1, T_{n}-T-1\right) \times S^{1}} \equiv 1 \text { and }|\nabla \eta| \leq C,
$$

by the elliptic estimates for the Dirac operator, we have

$$
\begin{aligned}
\left\|\eta \psi_{n}\right\|_{W^{1, \frac{4}{3}}\left(\mathbb{R}^{1} \times S^{1}\right)} \leq & C\left\|\not \partial\left(\eta \psi_{n}\right)\right\|_{L^{\frac{4}{3}}\left(\mathbb{R}^{1} \times S^{1}\right)} \\
\leq & C\left\|\nabla \eta \cdot \psi_{n}\right\|_{L^{\frac{4}{3}}\left(\mathbb{R}^{1} \times S^{1}\right)}+C\left\|\eta \not \psi_{n}\right\|_{L^{\frac{4}{3}}\left(\mathbb{R}^{1} \times S^{1}\right)} \\
\leq & C\left\|\psi_{n}\right\|_{L^{4}\left(\left(-T_{n}+T,-T_{n}+T+1\right) \times S^{1} \cup\left(T_{n}-T-1, T_{n}-T\right) \times S^{1}\right)} \\
& +C\left\|d \phi_{n}\right\|_{L^{2}\left(\left(-T_{n}+T, T_{n}-T\right) \times S^{1}\right)}\left\|\psi_{n}\right\|_{L^{4}\left(\left(-T_{n}+T, T_{n}-T\right) \times S^{1}\right)},
\end{aligned}
$$

where we used the Hölder inequality in the last estimate. Then (2.20) follows immediately from (2.19) and (2.10). Thus, we proved the statement (1) of the lemma.

For the statement (2), similar to the argument in (2.16), we may obtain

$$
\begin{aligned}
\int_{P_{i}}\left|\nabla \phi_{n}\right|^{2} d t d \theta \leq & 2 \int_{P_{i}}\left|\frac{\partial \phi_{n}}{\partial \theta}\right|^{2} d t d \theta+L\left|\operatorname{Re} \alpha\left(\phi_{n}, \psi_{n}\right)\right| \\
& +C(N)\left(\int_{P_{i}}\left|\psi_{n}\right|^{4}\right)^{\frac{1}{2}}\left(\int_{P_{i}}\left|\frac{\partial \phi_{n}}{\partial \theta}\right|^{2}\right)^{\frac{1}{2}} \\
& +\left(\int_{P_{i}}\left|\psi_{n}\right|^{4}\right)^{\frac{1}{4}}\left(\int_{P_{i}}\left|\frac{\partial \psi_{n}}{\partial \theta}\right|^{\frac{4}{3}}\right)^{\frac{3}{4}} \\
\leq & C(N, L, \Lambda)\left(\left(\int_{P_{i}}\left|\frac{\partial \phi_{n}}{\partial \theta}\right|^{2}\right)^{\frac{1}{2}}+\left(\int_{P_{i}}\left|\frac{\partial \psi_{n}}{\partial \theta}\right|^{2}\right)^{\frac{1}{2}}\right)+\operatorname{LeC\alpha }\left(\phi_{n}, \psi_{n}\right) \mid
\end{aligned}
$$




$$
\begin{aligned}
\leq & \operatorname{Co}(n, T)\left(e^{-\frac{i}{2} L}+e^{-\frac{k_{n}-i}{2} L}\right) \\
& +L\left|\operatorname{Re} \alpha\left(\phi_{n}, \psi_{n}\right)\right| .
\end{aligned}
$$

where the last inequality follows from (2.14) and (2.15).

Combining this with Lemma 2.3, we get

$$
\begin{aligned}
\operatorname{Osc}_{P_{i}} \phi_{n} & \leq C \sqrt{E\left(\phi_{n} ; P_{i-1} \cup P_{i} \cup P_{i+1}\right)} \\
& \leq \operatorname{Co}(n, T)\left(e^{-\frac{i}{4} L}+e^{-\frac{k_{n}-i}{4} L}\right)+C \sqrt{L\left|\operatorname{Re} \alpha\left(\phi_{n}, \psi_{n}\right)\right|} .
\end{aligned}
$$

Therefore,

$$
\begin{aligned}
O S c_{\left[-T_{n}+T, T_{n}-T\right] \times S^{1} \phi_{n}} & \leq \sum_{i=0}^{k_{n}} \operatorname{Osc}_{P_{i}} \phi_{n} \\
& \leq \sum_{i=0}^{k_{n}} \operatorname{Co}(n, T)\left(e^{-\frac{i}{4} L}+e^{-\frac{k_{n}-i}{4} L}\right)+\sqrt{L\left|\operatorname{Re} \alpha\left(\phi_{n}, \psi_{n}\right)\right|} T_{n} \\
& \leq \operatorname{Co}(n, T)+C \sqrt{\left|\operatorname{Re} \alpha\left(\phi_{n}, \psi_{n}\right)\right|} T_{n} .
\end{aligned}
$$

Then the conclusion of statement (2) follows immediately and we finished the proof of this lemma.

\section{Convergence to geodesics}

In this section, we will prove our main Theorem 1.2.

In fact, in [28], it was shown that the energy concentration at the nodes can be reduced to the study of the asymptotic behaviour of a sequence of Dirac-harmonic maps from long cylinders. Therefore, we just need to study the following problem:

Let $P_{n}=\left[-T_{n}, T_{n}\right] \times S^{1}$ with standard metric $d s^{2}=d t^{2}+d \theta^{2}$ and $T_{n} \rightarrow \infty$ as $n \rightarrow \infty$. Given a sequence of Dirac-harmonic maps $\left(\phi_{n}, \psi_{n}\right)$ from $P_{n}$ to $N$ with uniformly bounded energy $E\left(\phi_{n}, \psi_{n}\right) \leq \Lambda<\infty$, which satisfies

$$
\left(\phi_{n}\left(t-T_{n}, \theta\right), \psi_{n}\left(t-T_{n}, \theta\right)\right) \rightarrow\left(\omega^{-}, \zeta^{-}\right) \text {in } C^{\infty}\left([0, \infty) \times S^{1}\right),
$$

and

$$
\left(\phi_{n}\left(t+T_{n}, \theta\right), \psi_{n}\left(t+T_{n}, \theta\right)\right) \rightarrow\left(\omega^{+}, \zeta^{+}\right) \text {in } C^{\infty}\left((-\infty, 0] \times S^{1}\right),
$$

does $\phi_{n}$ converges to a geodesic? If so, how to compute the lengths of these curves?

Denote

$$
\mu:=\lim _{n \rightarrow \infty} \sqrt{\left|\operatorname{Re} \alpha\left(\phi_{n}, \psi_{n}\right)\right|} T_{n} .
$$

Now, we state the main theorem in this section.

Theorem 3.1 Let $N$ be a compact Riemannian manifold. Let $\left(\phi_{n}, \psi_{n}\right):\left[-T_{n}, T_{n}\right] \times S^{1} \rightarrow N$ be a sequence of Dirac-harmonic maps with uniformly bounded energy

$$
E\left(\phi_{n}, \psi_{n} ;\left[-T_{n}, T_{n}\right] \times S^{1}\right) \leq \Lambda,
$$


where $\left[-T_{n}, T_{n}\right] \times S^{1}$ is a cylinder with standard flat metric $d s^{2}=d t^{2}+d \theta^{2}$ and $T_{n} \rightarrow \infty$ as $n \rightarrow \infty$. Suppose that

$$
\left(\phi_{n}\left(t-T_{n}, \theta\right), \psi_{n}\left(t-T_{n}, \theta\right)\right) \rightarrow\left(\omega^{-}, \zeta^{-}\right) \text {in } C^{\infty}\left([0, \infty) \times S^{1}\right),
$$

and

$$
\left(\phi_{n}\left(t+T_{n}, \theta\right), \psi_{n}\left(t+T_{n}, \theta\right)\right) \rightarrow\left(\omega^{+}, \zeta^{+}\right) \text {in } C^{\infty}\left((-\infty, 0] \times S^{1}\right)
$$

if there is no energy concentration for $\left(\phi_{n}, \psi_{n}\right)$, then we have

(1) when $0 \leq \mu<\infty, \phi_{n}$ converges to a geodesic of length $\sqrt{\frac{2}{\pi}} \mu$;

(2) when $\mu=\infty$, the neck contains at least an infinite length geodesic.

To show Theorem 3.1, we shall first prove some lemmas.

Lemma 3.2 Under the assumption of Theorem 3.1 , let $\lambda \in(0,1)$ and

$$
\mu=\lim _{n \rightarrow \infty} \sqrt{\left|\operatorname{Re} \alpha\left(\phi_{n}, \psi_{n}\right)\right|} T_{n}>0,
$$

then for any $t_{n} \in\left[-\lambda T_{n}, \lambda T_{n}\right]$ and $k>0$, we have

$$
\lim _{n \rightarrow \infty} \frac{1}{\left|\operatorname{Re} \alpha\left(\phi_{n}, \psi_{n}\right)\right|}\left(\int_{Q_{t_{n}}(k)}\left|\frac{\partial \phi_{n}}{\partial \theta}\right|^{2} d t d \theta+\int_{Q_{t_{n}}(k)}\left|\frac{\partial \psi_{n}}{\partial \theta}\right|^{2} d t d \theta\right)=0 .
$$

Proof By Lemma 2.7, we have

$$
\begin{aligned}
& \left(\int_{Q_{t_{n}}(k)}\left|\frac{\partial \phi_{n}}{\partial \theta}\right|^{2} d t d \theta\right)^{\frac{1}{2}}+\left(\int_{Q_{t_{n}}(k)}\left|\frac{\partial \psi_{n}}{\partial \theta}\right|^{2} d t d \theta\right)^{\frac{1}{2}} \\
& \leq C\left(e^{-\frac{t_{n}-k-\left(-T_{n}+T\right)}{2}}+e^{-\frac{T_{n}-T-\left(t_{n}+k\right)}{2}}\right) o(n, T) \\
& \leq C e^{-\frac{(1-\lambda) T_{n}-k-T}{2}} o(n, T) .
\end{aligned}
$$

Since $\mu>0$, when $n$ is big enough, there holds

$$
\frac{1}{\sqrt{\left|\operatorname{Re} \alpha\left(\phi_{n}, \psi_{n}\right)\right|}} \leq C T_{n}
$$

Thus,

$$
\begin{aligned}
& \frac{1}{\left|\operatorname{Re} \alpha\left(\phi_{n}, \psi_{n}\right)\right|}\left(\int_{Q_{t_{n}}(k)}\left|\frac{\partial \phi_{n}}{\partial \theta}\right|^{2} d t d \theta+\int_{Q_{t_{n}}(k)}\left|\frac{\partial \psi_{n}}{\partial \theta}\right|^{2} d t d \theta\right) \\
& \leq C e^{-\left((1-\lambda) T_{n}-k-T\right)} T_{n}^{2} o(n, T) .
\end{aligned}
$$

Letting $n \rightarrow \infty$ and then $T \rightarrow \infty$, we will get the conclusion of the lemma.

From the above proof, it is easy to see that a stronger property holds. 
Remark 3.3 Under the assumption of Lemma 3.2, there holds

$$
\lim _{n \rightarrow \infty} \frac{1}{\left(\left|\operatorname{Re} \alpha\left(\phi_{n}, \psi_{n}\right)\right|\right)^{r}}\left(\int_{Q_{t_{n}}(k)}\left|\frac{\partial \phi_{n}}{\partial \theta}\right|^{2} d t d \theta+\int_{Q_{t_{n}}(k)}\left|\frac{\partial \psi_{n}}{\partial \theta}\right|^{2} d t d \theta\right)=0,
$$

for any positive integer $r$.

Next, we will show that there is no concentration for some stronger energy norm of the spinor part. The proof is based on applying some Hardy-type inequality on $\mathbb{R}^{2}$, as was done in $[11,15]$.

Lemma 3.4 Under the assumption of Theorem 3.1, there holds

$$
\lim _{T \rightarrow \infty} \lim _{n \rightarrow \infty} \int_{\left[-T_{n}+T, T_{n}-T\right] \times S^{1}}\left|\psi_{n}\right|^{2} d t d \theta=0 .
$$

Proof The key of the proof is the Hardy-type inequality on $\mathbb{R}^{2}$ that for any $f \in C_{0}^{\infty}\left(\mathbb{R}^{2} \backslash\{0\}\right)$, there holds

$$
\left\|\frac{f}{|x|}\right\|_{L^{1}\left(\mathbb{R}^{2}\right)} \leq\|\nabla f\|_{L^{1}\left(\mathbb{R}^{2}\right)}
$$

where the constant 1 is the best possible constant (for a simple proof, see [1]).

Firstly, we introduce a new coordinate system. Let $(r, \theta)$ be polar coordinates centered at 0 . Let $F: \mathbb{R}^{1} \times S^{1} \rightarrow \mathbb{R}^{2}, F(t, \theta)=\left(e^{t}, \theta\right)(t, \theta) \in \mathbb{R}^{1} \times S^{1}$ where $\mathbb{R}^{1} \times S^{1}$ is equipped with the metric $g=d t^{2}+d \theta^{2}$, which is conformal to the standard Euclidean metric $d s^{2}$ on $\mathbb{R}^{2}$. In fact,

$$
\left(F^{-1}\right)^{*} g=\frac{1}{r^{2}} d s^{2} .
$$

For convenience, we will respectively denote

$$
\Phi_{n}:=\phi_{n} \circ F^{-1} \text { and } \Psi_{n}:=e^{\frac{t}{2}} \psi_{n} \circ F^{-1} .
$$

Denoting $T_{0}:=-T_{n}+T, T_{1}:=T_{n}-T$, then $D_{e^{T_{n}-T}} \backslash D_{e^{-T_{n}+T}}$ changes to $\Sigma:=\left[T_{0}, T_{1}\right] \times S^{1}$ and $\left(\Phi_{n}, \Psi_{n}\right): D_{e^{T_{n}-T}} \backslash D_{e^{-T_{n}+T}} \rightarrow N$ is also a Dirac-harmonic map.

We choose the cut-off function $\eta \in C_{0}^{\infty}\left(D_{e^{T_{n}-T}} \backslash D_{e^{-T_{n}+T}}\right)$ such that $0 \leq \eta \leq 1$ and $\eta \equiv 1$ on $D_{e^{T_{n}-T-1}} \backslash D_{e^{-T_{n}+T+1}}$ and

$$
\begin{aligned}
& |\nabla \eta| \leq \frac{C}{e^{T_{n}-T}} \quad \text { on } \quad D_{e^{T_{n}-T}} \backslash D_{e^{T_{n}-T-1}} \quad \text { and } \\
& |\nabla \eta| \leq \frac{C}{e^{-T_{n}+T}} \quad \text { on } \quad D_{e^{-T_{n}+T+1}} \backslash D_{e^{-T_{n}+T}} .
\end{aligned}
$$

Taking $f=\eta\left|\Psi_{n}\right|^{2}$ in the inequality (3.5), we get

$$
\begin{aligned}
\left\|\eta \frac{\left|\Psi_{n}\right|^{2}}{|x|}\right\|_{L^{1}\left(\mathbb{R}^{2}\right)} & \leq\left\|\nabla\left(\eta\left|\Psi_{n}\right|^{2}\right)\right\|_{L^{1}\left(\mathbb{R}^{2}\right)} \\
& \leq\left\|2 \eta \Psi_{n} \nabla \Psi_{n}\right\|_{L^{1}\left(\mathbb{R}^{2}\right)}+\left\|\nabla \eta\left|\Psi_{n}\right|^{2}\right\|_{L^{1}\left(\mathbb{R}^{2}\right)} \\
& \leq\left\|2 \eta \Psi_{n} \frac{1}{|x|} \frac{\partial \Psi_{n}}{\partial \theta}\right\|_{L^{1}\left(\mathbb{R}^{2}\right)}+\left\|2 \eta \Psi_{n} \frac{\partial \Psi_{n}}{\partial r}\right\|_{L^{1}\left(\mathbb{R}^{2}\right)}+\left\|\nabla \eta\left|\Psi_{n}\right|^{2}\right\|_{L^{1}\left(\mathbb{R}^{2}\right)} .
\end{aligned}
$$

On the one hand, from the equation or spinor and conformal invariance, we know

$$
\frac{\partial \Psi_{n}}{\partial r}=\frac{\partial}{\partial r} \cdot \frac{1}{|x|} \frac{\partial}{\partial \theta} \cdot \frac{1}{|x|} \frac{\partial \Psi_{n}}{\partial \theta}+\frac{\partial}{\partial r} \cdot \mathcal{A}\left(d \Phi_{n}\left(e_{\gamma}\right), e_{\gamma} \cdot \Psi_{n}\right)
$$


So, we have

$$
\left|2 \eta \Psi_{n} \frac{\partial \Psi_{n}}{\partial r}\right| \leq\left|2 \eta \Psi_{n} \frac{1}{|x|} \frac{\partial \Psi_{n}}{\partial \theta}\right|+C|\eta|\left|d \Phi_{n}\right|\left|\Psi_{n}\right|^{2} .
$$

On the other hand, by inequality (2.11), we have

$$
|x|\left|d \Phi_{n}\right|+\sqrt{|x|}\left|\Psi_{n}\right| \leq C \epsilon \quad \text { on } \quad D_{e^{T_{n}-T}} \backslash D_{e^{-T_{n}+T}} .
$$

Combining these, we get

$$
\begin{aligned}
\left\|\eta \frac{\left|\Psi_{n}\right|^{2}}{|x|}\right\|_{L^{1}\left(\mathbb{R}^{2}\right)} & \leq 4\left\|\eta \Psi_{n} \frac{1}{|x|} \frac{\partial \Psi_{n}}{\partial \theta}\right\|_{L^{1}\left(\mathbb{R}^{2}\right)}+C\left\|\eta\left|d \Phi_{n}\right|\left|\Psi_{n}\right|^{2}\right\|_{L^{1}\left(\mathbb{R}^{2}\right)}+\left\|\nabla \eta\left|\Psi_{n}\right|^{2}\right\|_{L^{1}\left(\mathbb{R}^{2}\right)} \\
& \leq 4\left\|\eta \Psi_{n} \frac{1}{|x|} \frac{\partial \Psi_{n}}{\partial \theta}\right\|_{L^{1}\left(\mathbb{R}^{2}\right)}+C \epsilon\left\|\eta \frac{\left|\Psi_{n}\right|^{2}}{|x|}\right\|_{L^{1}\left(\mathbb{R}^{2}\right)}+\left\|\nabla \eta\left|\Psi_{n}\right|^{2}\right\|_{L^{1}\left(\mathbb{R}^{2}\right)} .
\end{aligned}
$$

Since we can take $\epsilon$ sufficiently small, we then have

$$
\begin{aligned}
& \left\|\eta \frac{\left|\Psi_{n}\right|^{2}}{|x|}\right\|_{L^{1}\left(\mathbb{R}^{2}\right)} \leq C\left\|\psi_{n} \frac{1}{|x|} \frac{\partial \Psi_{n}}{\partial \theta}\right\|_{L^{1}\left(D_{e^{T_{n}-T} \backslash D_{\left.e^{-T_{n}+T}\right)}}\right.} \\
& +C\left\|\nabla \eta\left|\Psi_{n}\right|^{2}\right\|_{L^{1}\left(D_{e^{T_{n}-T}} \backslash D_{\left.e^{-T_{n}+T}\right)}\right.}
\end{aligned}
$$

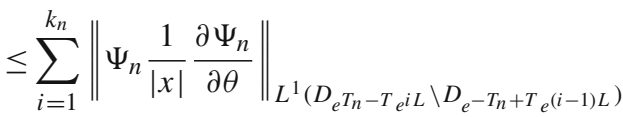

$$
\begin{aligned}
& +C\left\|\nabla \eta\left|\Psi_{n}\right|^{2}\right\|_{L^{1}\left(D_{e^{T_{n}-T}} \backslash D_{e^{-T_{n}}+T}\right)}
\end{aligned}
$$

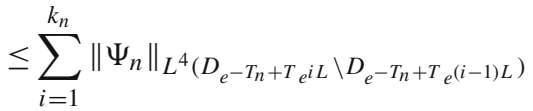

$$
\begin{aligned}
& \left\|\frac{1}{|x|} \frac{\partial \Psi_{n}}{\partial \theta}\right\|_{L^{\frac{4}{3}}\left(D_{e^{-T}+T_{n}} e^{i L} \backslash D_{\left.e^{-T_{n}+T} e^{(i-1) L}\right)}\right.} \\
& +C\left\|\nabla \eta\left|\Psi_{n}\right|^{2}\right\|_{L^{1}\left(D_{e^{T_{n}-T}} \backslash D_{\left.e^{-T_{n}+T}\right)}\right.}
\end{aligned}
$$

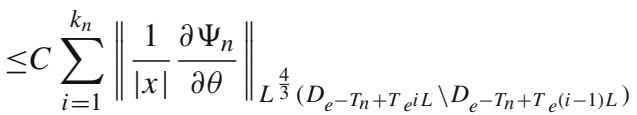

$$
\begin{aligned}
& +C\left\|\nabla \eta\left|\Psi_{n}\right|^{2}\right\|_{L^{1}\left(D_{e^{T_{n}-T}} \backslash D_{e^{-T_{n}+T}}\right)} \\
& \leq C \sum_{i=1}^{k_{n}}\left\|\frac{\partial \psi_{n}}{\partial \theta}\right\|_{L^{\frac{4}{3}\left(P_{i}\right)}}+C e^{-T_{n}+T}\left\|\left|\Psi_{n}\right|^{2}\right\|_{L^{1}\left(D_{e^{T_{n}-T}} \backslash D_{e^{T_{n}-T-1}}\right)}
\end{aligned}
$$

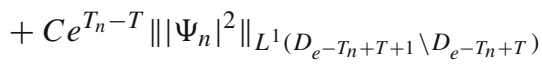

$$
\begin{aligned}
& \leq C \epsilon \sum_{i=1}^{k_{n}}\left(e^{-\frac{i}{2} L}+e^{-\frac{k_{\alpha}-i}{2} L}\right)+C\left\|\Psi_{n}\right\|_{L^{4}\left(D_{e^{T_{n}}-T} \backslash D_{e^{T_{n}-T-1}}\right)}^{2}
\end{aligned}
$$

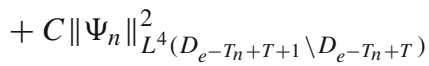

$$
\begin{aligned}
& \leq C \epsilon+C\left\|\psi_{n}\right\|_{L^{4}\left(P_{k_{n}}\right)}^{2}+C\left\|\psi_{n}\right\|_{L^{4}\left(P_{1}\right)}^{2} \leq C \epsilon
\end{aligned}
$$


where we used (2.10) and (2.15).

Thus,

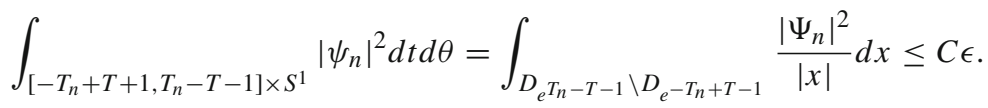

Combining this with (2.10) again, we have

$$
\int_{\left[-T_{n}+T, T_{n}-T\right] \times S^{1}}\left|\psi_{n}\right|^{2} d t d \theta \leq C \epsilon .
$$

Combing Lemma 3.4 with the Hardy type inequality (3.5), we shall derive the exponential decay of the energy of spinor part on the neck region.

Lemma 3.5 Under the assumption of Theorem 3.1, then we have

$$
\int_{P_{i}}\left|\psi_{n}\right|^{2} d t d \theta \leq\left(e^{-\frac{i L}{4}}+e^{-\frac{\left(k_{n}-i\right) L}{4}}\right) o(n, T),
$$

which implies

$$
\int_{P_{i}}\left|\psi_{n}\right|^{4} d t d \theta \leq\left(e^{-\frac{i L}{4}}+e^{-\frac{\left(k_{n}-i\right) L}{4}}\right) o(n, T) .
$$

Proof Define

$$
f_{1}(t):=\int_{D_{e^{\left(t_{0}+t\right) L} L_{e}-T_{n}+T} \backslash D_{e^{\left(t_{0}-t\right) L_{e}-T_{n}+T}}} \frac{\left|\Psi_{n}\right|^{2}}{|x|} d x
$$

for any $t_{0} \in\left(1, k_{n}\right)$ and $0 \leq t \leq \min \left\{t_{0}, k_{n}-t_{0}\right\}$.

For any $\rho>0$, taking the cut-off function $\eta \in C_{0}^{\infty}\left(D_{e^{\left(t_{0}+t\right) L} e^{-T_{n}+T}+\rho} \backslash D_{e^{\left(t_{0}-t\right) L}} e^{-T_{n}+T}-\rho\right)$

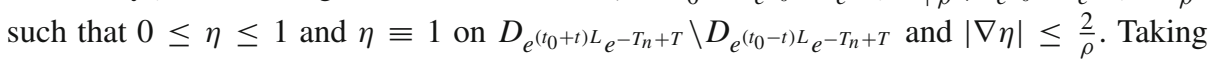
$f=\eta\left|\Psi_{n}\right|^{2}$ in the Hardy inequality (3.5) and by (3.8), we get

$$
\left\|\eta \frac{\left|\Psi_{n}\right|^{2}}{|x|}\right\|_{L^{1}\left(\mathbb{R}^{2}\right)} \leq 4\left\|\eta \Psi_{n} \frac{1}{|x|} \frac{\partial \Psi_{n}}{\partial \theta}\right\|_{L^{1}\left(\mathbb{R}^{2}\right)}+C \epsilon\left\|\eta \frac{\left|\Psi_{n}\right|^{2}}{|x|}\right\|_{L^{1}\left(\mathbb{R}^{2}\right)}+\left\|\nabla \eta\left|\Psi_{n}\right|^{2}\right\|_{L^{1}\left(\mathbb{R}^{2}\right)} .
$$

Taking $\epsilon>0$ sufficiently small such that $C \epsilon \leq \frac{1}{2}$, we have

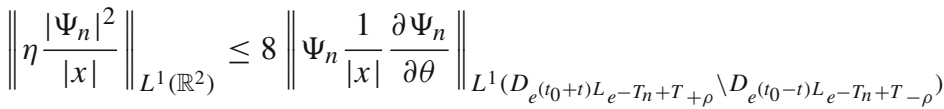

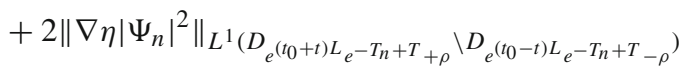

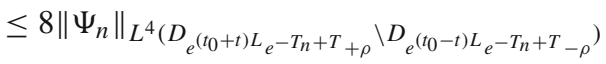

$$
\begin{aligned}
& \left\||x|^{-1} \frac{\partial \Psi_{n}}{\partial \theta}\right\|_{L^{\frac{4}{3}}\left(D_{e^{\left(t_{0}+t\right) L} e^{-T_{n}+T}+\rho} \backslash D_{e^{\left(t_{0}-t\right) L} e^{-T_{n}+T}-\rho}\right)} \\
& +\frac{4}{\rho}\left\|\left|\Psi_{n}\right|^{2}\right\|_{L^{1}\left(D_{e^{\left(t_{0}+t\right) L} L_{e}-T_{n}+T} \backslash \rho\right.} \backslash D_{\left.e^{\left(t_{0}+t\right) L} e^{-T_{n}+T}\right)}
\end{aligned}
$$

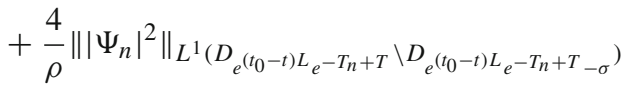




$$
\begin{aligned}
& \leq C\left\|\frac{\partial \psi_{n}}{\partial \theta}\right\|_{L^{\frac{4}{3}}\left(Q_{-T_{n}+T+t_{0} L}(t L+1)\right)}
\end{aligned}
$$

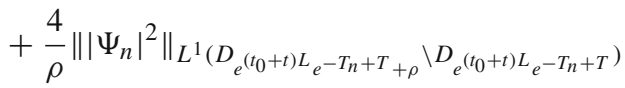

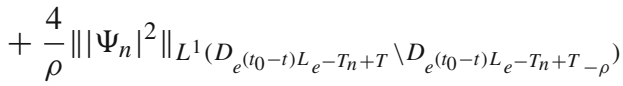

$$
\begin{aligned}
& \leq\left(e^{-\frac{1}{2}\left(t_{0}-t\right) L}+e^{-\frac{1}{2}\left(k_{n}-t_{0}-t\right) L}\right) o(n, T)
\end{aligned}
$$

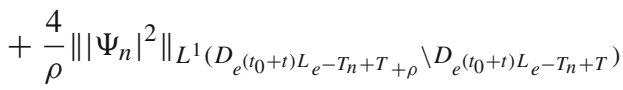

$$
\begin{aligned}
& +\frac{4}{\rho}\left\|\left|\Psi_{n}\right|^{2}\right\|_{L^{1}\left(D_{e^{\left(t_{0}-t\right) L} e^{-T_{n}+T}} \backslash D_{e^{\left(t_{0}-t\right) L_{e}-T_{n}+T_{-}}}\right)}
\end{aligned}
$$

where $\rho$ is small and the last inequality is from Lemma 2.7.

Letting $\rho \rightarrow 0$, we get

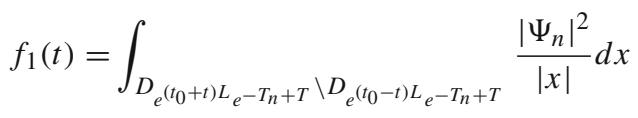

$$
\begin{aligned}
& \leq\left(e^{-\frac{1}{2}\left(t_{0}-t\right) L}+e^{-\frac{1}{2}\left(k_{\alpha}-t_{0}-t\right) L}\right) o(n, T)+4 e^{\left(t_{0}+t\right) L} \lambda_{\alpha} R \int_{\partial D_{e^{\left(t_{0}+t\right) L} e^{-T_{n}+T}}} \frac{\left|\Psi_{n}\right|^{2}}{|x|} \\
& +4 e^{\left(t_{0}-t\right) L} e^{-T_{n}+T} \int_{\partial D_{e^{\left(t_{0}-t\right) L} e^{-T_{n}+T}}} \frac{\left|\Psi_{n}\right|^{2}}{|x|} \\
& \leq\left(e^{-\frac{1}{2}\left(t_{0}-t\right) L}+e^{-\frac{1}{2}\left(k_{n}-t_{0}-t\right) L}\right) o(n, T)+\frac{4}{L} f_{1}^{\prime}(t) .
\end{aligned}
$$

This is

$$
\begin{aligned}
\left(e^{-\frac{L}{4} t} f_{1}(t)\right)^{\prime} & \geq-e^{-\frac{L}{4} t}\left(e^{-\frac{1}{2}\left(t_{0}-t\right) L}+e^{-\frac{1}{2}\left(k_{n}-t_{0}-t\right) L}\right) o(n, T) \\
& =-e^{\frac{L}{4} t}\left(e^{-\frac{1}{2} t_{0} L}+e^{-\frac{1}{2}\left(k_{n}-t_{0}\right) L}\right) o(n, T) .
\end{aligned}
$$

Without loss of generality, we may assume $t_{0} \leq k_{n}-t_{0}$. Then, integrating the above differential inequality from 1 to $t_{0}$, we get

$$
f_{1}(1) \leq C e^{-\frac{L}{4} t_{0}} f_{1}\left(t_{0}\right)+o(n, T) e^{-\frac{L}{2} t_{0}} \int_{1}^{t_{0}} e^{\frac{L}{4} t} d t=e^{-\frac{L}{4} t_{0}} o(n, T)
$$

where the second equality follows from Lemma 3.4 since

$$
f_{1}\left(t_{0}\right) \leq \int_{D_{e^{T_{n}-T} \backslash D_{e^{-T_{n}+T}}}} \frac{\left|\Psi_{n}\right|^{2}}{|x|} d x=\int_{\left[-T_{n}+T, T_{n}-T\right] \times S^{1}}\left|\psi_{n}\right|^{2} d t d \theta .
$$

In the case of $k_{\alpha}-t_{0} \leq t_{0}$, we can apply similar arguments to get

$$
f_{1}(1) \leq e^{-\frac{L}{4}\left(k_{n}-t_{0}\right)} o(n, T) .
$$

Thus, we have proved (3.9). The estimate (3.10) is a consequence of (3.9) and (2.11). 
Lemma 3.6 Under the assumption of Theorem 3.1, let $\mu>0$ and $\lambda \in(0,1)$, then there hold

$$
\begin{aligned}
& \left\|\frac{1}{\sqrt{\left|\operatorname{Re} \alpha\left(\phi_{n}, \psi_{n}\right)\right|}}\left(\phi_{n}-\frac{1}{2 \pi} \int_{0}^{2 \pi} \phi_{n} d \theta\right)\right\|_{C^{1}\left(\left[-\lambda T_{n}, \lambda T_{n}\right] \times S^{1}\right)} \rightarrow 0, \\
& \left\|\frac{\psi_{n}}{\left|\operatorname{Re} \alpha\left(\phi_{n}, \psi_{n}\right)\right|}\right\|_{C^{0}\left(\left[-\lambda T_{n}, \lambda T_{n}\right] \times S^{1}\right)}^{\rightarrow} \rightarrow 0 .
\end{aligned}
$$

Proof We first prove (3.14). In fact, for any $t \in\left[-\lambda T_{n}, \lambda T_{n}\right] \times S^{1}$ and $k>0$, by Lemmas 2.3 and 3.5, we have

$$
\begin{aligned}
\left\|\psi_{n}\right\|_{C^{0}\left(Q_{t}(k)\right)} \leq C(N)\left\|\psi_{n}\right\|_{L^{4}\left(Q_{t}(2 k)\right)} & \leq\left(e^{-\frac{t-T_{n}}{16}}+e^{-\frac{T_{n}-t}{16}}\right) o(n, T) \\
& \leq e^{-\frac{(1-\lambda) T_{n}}{16}} o(n, T) .
\end{aligned}
$$

Noting that $\mu>0$, when $n$ is big enough, there holds

$$
\frac{1}{T_{n}} \leq C \sqrt{\left|\operatorname{Re} \alpha\left(\phi_{n}, \psi_{n}\right)\right|}
$$

Then, we get

$$
\frac{\left\|\psi_{n}\right\|_{C^{0}\left(Q_{t}(k)\right)}}{\left|\operatorname{Re} \alpha\left(\phi_{n}, \psi_{n}\right)\right|} \leq e^{-\frac{(1-\lambda) T_{n}}{16}} T_{n}^{2} o(n, T)
$$

which implies (3.14) since $\lambda \in(0,1)$.

For (3.13), we prove it by a contradiction argument which is similar to the proof of Lemma 2.7 in [7].

If it was false, then there would exist $t_{n} \in\left[-\lambda T_{n}, \lambda T_{n}\right]$ and $\theta_{n} \in[0,2 \pi]$ such that

$$
\frac{1}{\sqrt{\left|\operatorname{Re} \alpha\left(\phi_{n}, \psi_{n}\right)\right|}}\left(\left|\nabla \phi_{n}\left(t_{n}, \theta_{n}\right)-\nabla \phi_{n}^{*}\left(t_{n}\right)\right|+\left|\phi_{n}\left(t_{n}, \theta_{n}\right)-\phi_{n}^{*}\left(t_{n}\right)\right|\right) \rightarrow b \neq 0 .
$$

By (2.21), for any $t \in\left[-\lambda T_{n}, \lambda T_{n}\right] \times S^{1}$ and $k>0$, when $n$ is big enough, we have

$$
\begin{aligned}
\int_{Q_{t}(k)}\left|\nabla \phi_{n}\right|^{2} d t d \theta & \leq C(k)\left(e^{-\frac{t+T_{n}}{2}}+e^{-\frac{T_{n}-t}{2}}\right) o(n, T)+2 k\left|\operatorname{Re} \alpha\left(\phi_{n}, \psi_{n}\right)\right| \\
& \leq C(k) e^{-\frac{(1-\lambda) T_{n}}{4}} o(n, T)+2 k\left|\operatorname{Re} \alpha\left(\phi_{n}, \psi_{n}\right)\right| .
\end{aligned}
$$

Combining this with (3.15) and (3.17), since $\lambda \in(0,1)$, when $n$ is sufficiently large, we get

$$
\int_{Q_{t}(2 k)}\left|\nabla \phi_{n}\right|^{2} d t d \theta \leq C(k)\left|\operatorname{Re} \alpha\left(\phi_{n}, \psi_{n}\right)\right| .
$$

By small energy regularity Lemma 2.3, we obtain that for any $\beta \in(0,1)$, there holds

$$
\left\|\nabla \phi_{n}\right\|_{C^{\beta}\left(Q_{t}(k)\right)} \leq C(\beta, k) \sqrt{\left|\operatorname{Re} \alpha\left(\phi_{n}, \psi_{n}\right)\right|} .
$$

Setting

$$
u_{n}:=\frac{1}{\sqrt{\left|\operatorname{Re} \alpha\left(\phi_{n}, \psi_{n}\right)\right|}}\left(\phi_{n}\left(t_{n}+t, \theta\right)-\phi_{n}\left(t_{n}, 0\right)\right),
$$

then from (3.19), it is easy to see that

$$
\left\|\nabla u_{n}\right\|_{C^{\beta}\left(Q_{0}(k)\right)} \leq C(\beta, k) .
$$


Combining this with the fact that $u_{n}(0,0)=0$, we have

$$
\left\|u_{n}\right\|_{C^{1, \beta}\left(Q_{0}(k)\right)} \leq C(\beta, k) .
$$

Noting that $\left|\psi_{n}\right| \leq C\left|\operatorname{Re} \alpha\left(\phi_{n}, \psi_{n}\right)\right|$, it is easy to prove that $u_{n}$ satisfies the following equation

$$
\Delta u_{n}+\sqrt{\left|\operatorname{Re} \alpha\left(\phi_{n}, \psi_{n}\right)\right|}(A(y)+o(1))\left(\nabla u_{n}, \nabla u_{n}\right)+\left|\operatorname{Re} \alpha\left(\phi_{n}, \psi_{n}\right)\right|^{2} O\left(\left|\nabla u_{n}\right|\right)=0 .
$$

Therefore, we know that after passing to a subsequence, there holds

$$
u_{n} \rightarrow u \in C_{l o c}^{1}\left(\mathbb{R}^{1} \times S^{1}\right)
$$

where $u$ is a harmonic function, i.e. $\Delta u=0$.

Moreover, by Lemma 3.2, we get

$$
\frac{\partial u}{\partial \theta}=0
$$

Thus, we know that the harmonic function $u$ must be of the form

$$
u(t)=\vec{a} t,
$$

where $\vec{a}=\left(a^{1}, \ldots, a^{K}\right) \in T_{y} N$. Let $\lim _{n \rightarrow \infty} \theta_{n}=\theta_{0}$, then it is obvious that

$$
\left|\nabla u\left(0, \theta_{0}\right)-\nabla u^{*}(0)\right|+\left|u\left(0, \theta_{0}\right)-u^{*}(0)\right|=0 .
$$

However, by (3.16), we have

$$
\begin{aligned}
b & =\lim _{n \rightarrow \infty} \frac{1}{\sqrt{\left|\operatorname{Re} \alpha\left(\phi_{n}, \psi_{n}\right)\right|}}\left(\left|\nabla \phi_{n}\left(t_{n}, \theta_{n}\right)-\nabla \phi_{n}^{*}\left(t_{n}\right)\right|+\left|\phi_{n}\left(t_{n}, \theta_{n}\right)-\phi_{n}^{*}\left(t_{n}\right)\right|\right) \\
& =\lim _{n \rightarrow \infty}\left|\nabla u_{n}\left(0, \theta_{n}\right)-\nabla u_{n}^{*}(0)\right|+\lim _{n \rightarrow \infty}\left|u_{n}\left(0, \theta_{n}\right)-u_{n}^{*}(0)\right| \\
& =\left|\nabla u\left(0, \theta_{0}\right)-\nabla u^{*}(0)\right|+\left|u\left(0, \theta_{0}\right)-u^{*}(0)\right|=0,
\end{aligned}
$$

which is a contradiction. So, (3.13) holds and we finished the proof of the lemma.

Lemma 3.7 Under the assumptions of Theorem 3.1, let $\mu>0$ and $\lambda \in(0,1)$, then there holds

$$
\left\|\frac{1}{\sqrt{\left|\operatorname{Re} \alpha\left(\phi_{n}, \psi_{n}\right)\right|}}\left|\nabla \phi_{n}\right|-\frac{1}{\sqrt{2 \pi}}\right\|_{C^{0}\left(\left[-\lambda T_{n}, \lambda T_{n}\right] \times S^{1}\right)} \rightarrow 0 .
$$

Proof In fact, if (3.21) does not hold, then there exist $t_{n} \in\left[-\lambda T_{n}, \lambda T_{n}\right]$ and $\theta_{n} \in[0,2 \pi]$ such that

$$
\frac{1}{\sqrt{\left|\operatorname{Re} \alpha\left(\phi_{n}, \psi_{n}\right)\right|}}\left|\nabla \phi_{n}\left(t_{n}, \theta_{n}\right)\right| \rightarrow d \neq \frac{1}{\sqrt{2 \pi}} .
$$

By similar argument as in the proof of Lemma 3.6, we may assume

$$
u_{n}:=\frac{1}{\sqrt{\left|\operatorname{Re} \alpha\left(\phi_{n}, \psi_{n}\right)\right|}}\left(\phi_{n}\left(t_{n}+t, \theta\right)-\phi_{n}\left(t_{n}, 0\right)\right) \rightarrow u(t) \text { in } C_{l o c}^{1}\left(\mathbb{R}^{1} \times S^{1}\right),
$$

where

$$
u(t)=\vec{a} t
$$


with $|\vec{a}|=d$. Then,

$$
\lim _{n \rightarrow \infty} \int_{[-1,1] \times S^{1}}\left|\nabla u_{n}\right|^{2} d t d \theta=4 \pi|\vec{a}|^{2} .
$$

On the other hand, by Lemmas 2.1 and 3.2, we have

$$
\begin{aligned}
& \lim _{n \rightarrow \infty} \int_{[-1,1] \times S^{1}}\left|\nabla u_{n}\right|^{2} d t d \theta \\
& \quad=\lim _{n \rightarrow \infty} \frac{1}{\left|\operatorname{Re} \alpha\left(\phi_{n}, \psi_{n}\right)\right|} \int_{Q_{t_{n}}(1)}\left|\nabla \phi_{n}\right|^{2} d t d \theta \\
& =\lim _{n \rightarrow \infty} \frac{1}{\left|\operatorname{Re} \alpha\left(\phi_{n}, \psi_{n}\right)\right|} \int_{Q_{t_{n}(1)}}\left(\left|\frac{\partial \phi_{n}}{\partial t}\right|^{2}-\left|\frac{\partial \phi_{n}}{\partial \theta}\right|^{2}-\operatorname{Re}\left\langle\psi_{n}, \frac{\partial}{\partial \theta} \cdot \widetilde{\nabla}_{\frac{\partial}{\partial \theta}} \psi_{n}\right\rangle\right) d t d \theta \\
& =2 .
\end{aligned}
$$

Combining (3.23) with (3.22), we get

$$
d=|\vec{a}|=\frac{1}{\sqrt{2 \pi}}
$$

which is also a contradiction. Thus, (3.21) holds and we finished the proof of this lemma.

Now, with the help of the previous lemmas, we can prove Theorem 3.1.

Proof of Theorem 3.1 When $\mu=0$, then the conclusions of Theorem 3.1 follow immediately from Lemma 2.8. We just need to consider the case that $\mu>0$.

Denote

$$
\gamma_{n}: \quad \phi_{n}^{*}(t)=\frac{1}{2 \pi} \int_{0}^{2 \pi} \phi_{n}(t, \theta) d \theta
$$

where $t \in\left[-\lambda T_{n}, \lambda T_{n}\right], \lambda \in(0,1)$.

By Lemma 2.3, it is easy to see that $\gamma_{n}$ converges to some curve on $N$ denoted by $\gamma$. Next, we will show that $\gamma$ is just a geodesic on $N$.

Denoting

$$
\dot{\phi}_{n}^{*}=\frac{d \phi_{n}^{*}}{d t}, \ddot{\phi}_{n}^{*}=\frac{d^{2} \phi_{n}^{*}}{d t^{2}},
$$

by a direct computation, we have

$$
\begin{aligned}
\ddot{\phi}_{n}^{*}(t) & =\frac{1}{2 \pi} \int_{0}^{2 \pi} \frac{\partial^{2} \phi_{n}}{\partial t^{2}}(t, \theta) d \theta=\frac{1}{2 \pi} \int_{0}^{2 \pi} \Delta \phi_{n} d \theta \\
& =\frac{1}{2 \pi} \int_{0}^{2 \pi} A\left(\phi_{n}\right)\left(\nabla \phi_{n}, \nabla \phi_{n}\right) d \theta+\frac{1}{2 \pi} \int_{0}^{2 \pi} \operatorname{Re}\left(P\left(\mathcal{A}\left(d \phi_{n}\left(e_{\gamma}\right), e_{\gamma} \cdot \psi_{n}\right) ; \psi_{n}\right)\right) d \theta .
\end{aligned}
$$

Let $s$ be the arc length parameter of $\phi_{n}^{*}(t)$, i.e.

$$
s(t)=\int_{t_{0}}^{t}\left|\dot{\phi}_{n}^{*}(\sigma)\right| d \sigma,
$$


where $t_{0} \in\left[-\lambda T_{n}, \lambda T_{n}\right]$. Without loss of generality, we assume $s\left(t_{0}\right)=0$. By Lemmas 3.6 and 3.7, for any $\lambda \in(0,1)$, we have

$$
\begin{aligned}
\int_{t_{0}-\lambda T_{n}}^{t_{0}+\lambda T_{n}}\left|\dot{\phi}_{n}^{*}\right| d t & =2 \lambda T_{n} \sqrt{\left|\operatorname{Re} \alpha\left(\phi_{n}, \psi_{n}\right)\right|}\left(\frac{1}{\sqrt{2 \pi}}+o(1)\right) \\
& \rightarrow \frac{2 \lambda \mu}{\sqrt{2 \pi}}>0
\end{aligned}
$$

as $n \rightarrow \infty$. Thus, there exists $\lambda^{\prime} \in(\lambda, 1)$ such that

$$
\left.\phi_{n}^{*}\right|_{\left[s\left(t_{0}\right)-s, s\left(t_{0}\right)+s\right]} \subset \phi_{n}^{*}\left(\left[-\lambda^{\prime} T_{n}, \lambda^{\prime} T_{n}\right]\right)
$$

whenever $s \in\left(0, \frac{\lambda \mu}{\sqrt{2 \pi}}\right)$.

Computing directly, we obtain

$$
\begin{aligned}
\frac{d^{2} \phi_{n}^{*}}{d s^{2}}= & A_{\gamma_{n}}\left(\phi_{n}^{*}(s)\right)\left(\frac{d \phi_{n}^{*}}{d s}, \frac{d \phi_{n}^{*}}{d s}\right)=\frac{1}{\left|\dot{\phi}_{n}^{*}\right|^{2}} A_{\gamma_{n}}\left(\phi_{n}^{*}(t)\right)\left(\frac{d \phi_{n}^{*}}{d t}, \frac{d \phi_{n}^{*}}{d t}\right) \\
= & \frac{1}{\left|\dot{\phi}_{n}^{*}\right|^{2}}\left(\ddot{\phi}_{n}^{*}-\frac{\left\langle\ddot{\phi}_{n}^{*}, \dot{\phi}_{n}^{*}\right\rangle}{\left|\dot{\phi}_{n}^{*}\right|^{2}} \dot{\phi}_{n}^{*}\right) \\
= & \frac{1}{\left|\dot{\phi}_{n}^{*}\right|^{2}} \frac{1}{2 \pi}\left(\int_{0}^{2 \pi} A\left(\phi_{n}\right)\left(\nabla \phi_{n}, \nabla \phi_{n}\right) d \theta\right. \\
& \left.+\int_{0}^{2 \pi} \operatorname{Re}\left(P\left(\mathcal{A}\left(d \phi_{n}\left(e_{\gamma}\right), e_{\gamma} \cdot \psi_{n}\right) ; \psi_{n}\right)\right) d \theta\right) \\
& -\frac{\dot{\phi}_{n}^{*}}{\left|\dot{\phi}_{n}^{*}\right|^{4}} \frac{1}{2 \pi} \int_{0}^{2 \pi}\left\langle A\left(\phi_{n}\right)\left(\nabla \phi_{n}, \nabla \phi_{n}\right), \dot{\phi}_{n}^{*}\right\rangle d \theta \\
& -\frac{\dot{\phi}_{n}^{*}}{\left|\dot{\phi}_{n}^{*}\right|^{4}} \frac{1}{2 \pi} \int_{0}^{2 \pi}\left\langle\operatorname{Re}\left(P\left(\mathcal{A}\left(d \phi_{n}\left(e_{\gamma}\right), e_{\gamma} \cdot \psi_{n}\right) ; \psi_{n}\right)\right), \dot{\phi}_{n}^{*}\right\rangle d \theta \\
:= & \mathbf{I}+\mathbf{I I}+\mathbf{I I I}+\mathbf{I V} .
\end{aligned}
$$

On the one hand, by Lemmas 3.6, 3.7 and using the fact that

$$
\left\langle A\left(\phi_{n}\right)\left(\nabla \phi_{n}, \nabla \phi_{n}\right), \frac{\partial \phi_{n}}{\partial t}\right\rangle=0,
$$

we have

$$
\begin{aligned}
\mathbf{I I I} & =\frac{\dot{\phi}_{n}^{*}}{\left|\dot{\phi}_{n}^{*}\right|^{4}} \frac{1}{2 \pi} \int_{0}^{2 \pi}\left\langle A\left(\phi_{n}\right)\left(\nabla \phi_{n}, \nabla \phi_{n}\right), \dot{\phi}_{n}^{*}-\frac{\partial \phi_{n}}{\partial t}\right\rangle d \theta \\
& =\frac{\dot{\phi}_{n}^{*}}{\left|\dot{\phi}_{n}^{*}\right|^{4}}\left(\sqrt{\left|\operatorname{Re} \alpha\left(\phi_{n}, \psi_{n}\right)\right|}\right)^{3} o(1) \rightarrow 0
\end{aligned}
$$

as $n \rightarrow \infty$.

On the other hand, also from Lemmas 3.6 and 3.7, it is easy to see that

$$
\begin{aligned}
\mathbf{I I}+\mathbf{I V} & \leq C(N) \frac{1}{\left|\dot{\phi}_{n}^{*}\right|^{2}}\left\|\nabla \phi_{n}\right\|_{L^{\infty}\left(\left[-\lambda T_{n}, \lambda T_{n}\right] \times S^{1}\right)}\left\|\psi_{n}\right\|_{L^{\infty}\left(\left[-\lambda T_{n}, \lambda T_{n}\right] \times S^{1}\right)}^{2} \\
& \leq C(N)\left|\operatorname{Re} \alpha\left(\phi_{n}, \psi_{n}\right)\right|^{\frac{3}{2}} \rightarrow 0,
\end{aligned}
$$

as $n \rightarrow \infty$. 
Therefore, we obviously see that

$$
\left|\frac{d^{2} \phi_{n}^{*}}{d s^{2}}\right| \leq C(N) .
$$

Thus, $\phi_{n}^{*}(s)$ will converge locally to a smooth vector valued function from $[0, s]$ into $\mathbb{R}^{K}$, denoted by $\omega(s)$, in the sense of $C^{1}$, i.e. $\left.\gamma_{n}\right|_{\left[-\lambda T_{n}, \lambda T_{n}\right]}$ converges locally to the curve $\gamma$. Next, we will show that $\gamma$ is a geodesic.

By Lemmas 3.6 and 3.7, we obtain

$$
\begin{aligned}
\mathbf{I}= & \frac{1}{\left|\dot{\phi}_{n}^{*}\right|^{2}} \frac{1}{2 \pi} \int_{0}^{2 \pi} A\left(\phi_{n}\right)\left(\nabla \phi_{n}, \nabla \phi_{n}\right) d \theta \\
= & \frac{1}{\left|\dot{\phi}_{n}^{*}\right|^{2}} \frac{1}{2 \pi}\left(\int_{0}^{2 \pi}\left(A\left(\phi_{n}\right)-A\left(\phi_{n}^{*}\right)\right)\left(\nabla \phi_{n}, \nabla \phi_{n}\right)+A\left(\phi_{n}^{*}\right)\left(\nabla\left(\phi_{n}-\phi_{n}^{*}\right), \nabla \phi_{n}\right) d \theta\right) \\
& +\frac{1}{\left|\dot{\phi}_{n}^{*}\right|^{2}} \frac{1}{2 \pi} \int_{0}^{2 \pi} A\left(\phi_{n}^{*}\right)\left(\nabla \phi_{n}^{*}, \nabla\left(\phi_{n}-\phi_{n}^{*}\right)\right) d \theta+\frac{1}{\left|\dot{\phi}_{n}^{*}\right|^{2}} \frac{1}{2 \pi} \int_{0}^{2 \pi} A\left(\phi_{n}^{*}\right)\left(\nabla \phi_{n}^{*}, \nabla \phi_{n}^{*}\right) d \theta \\
= & \frac{1}{\left|\dot{\phi}_{n}^{*}\right|^{2}} A\left(\phi_{n}^{*}\right)\left(\nabla \phi_{n}^{*}, \nabla \phi_{n}^{*}\right)+\frac{\left|\operatorname{Re} \alpha\left(\phi_{n}, \psi_{n}\right)\right|}{\left|\dot{\phi}_{n}^{*}\right|^{2}} o(1) .
\end{aligned}
$$

Combining (3.24) with (3.25), (3.26) and (3.27), we have

$$
\frac{d \omega}{d s}(s)-\frac{d \omega}{d s}(0)=\int_{0}^{s} A(\omega)\left(\frac{d \omega}{d s}, \frac{d \omega}{d s}\right)
$$

which implies

$$
\frac{d^{2} \omega}{d s^{2}}=A(\omega)\left(\frac{d \omega}{d s}, \frac{d \omega}{d s}\right) .
$$

Therefore, we get that the Dirac-harmonic neck converges to a geodesic $\gamma$ on the target manifold $N$.

Finally, we compute the length of the geodesic. We consider the following two cases.

Case 1: $\mu<\infty$.

Suppose $i_{1} \in\left[1, k_{n}\right]$ is a positive integer such that

$$
-T_{n}+T+\left(i_{1}-1\right) L \leq-\lambda T_{n}<-T_{n}+T+i_{1} L .
$$

By (2.22), there holds

$$
\begin{aligned}
O S c_{\left[-T_{n}+T,-\lambda T_{n}\right] \times S^{1}} \phi_{n} & \leq \sum_{i=1}^{i_{1}} \operatorname{OSc}_{P_{i}} \phi_{n} \\
& \leq \operatorname{Co}(n, T) \sum_{i=1}^{i_{1}}\left(e^{-\frac{i}{4} L}+e^{-\frac{k_{n}-i}{4} L}\right)+C \sqrt{L\left|\operatorname{Re} \alpha\left(\phi_{n}, \psi_{n}\right)\right|} \\
& \leq \operatorname{Co}(n, T)+C \sqrt{\left|\operatorname{Re} \alpha\left(\phi_{n}, \psi_{n}\right)\right|}\left((1-\lambda) T_{n}-T\right) .
\end{aligned}
$$

Similarly,

$$
O s c_{\left[\lambda T_{n}, T_{n}-T\right] \times S^{1}} \phi_{n} \leq \operatorname{Co}(n, T)+\sqrt{\left|\operatorname{Re} \alpha\left(\phi_{n}, \psi_{n}\right)\right|}\left((1-\lambda) T_{n}-T\right) .
$$


Therefore, we get

$$
\lim _{\lambda \rightarrow 1} \lim _{T \rightarrow \infty} \lim _{n \rightarrow \infty}\left(O s c_{\left[-T_{n}+T,-\lambda T_{n}\right] \times S^{1}} \phi_{n}+O s c_{\left[\lambda T_{n}, T_{n}-T\right] \times S^{1}} \phi_{n}\right)=0 .
$$

Combining this with Lemma 3.6, the length of the limit geodesic is

$$
\begin{aligned}
L(\gamma) & =\lim _{\lambda \rightarrow 1} \lim _{n \rightarrow \infty} \int_{-\lambda T_{n}}^{\lambda T_{n}}\left|\dot{\phi}_{n}^{*}\right| d t \\
& =\lim _{\lambda \rightarrow 1} \lim _{n \rightarrow \infty} 2 \lambda T_{n} \sqrt{\left|\operatorname{Re} \alpha\left(\phi_{n}, \psi_{n}\right)\right|}\left(\frac{1}{\sqrt{2 \pi}}+o(1)\right)=\sqrt{\frac{2}{\pi}} \mu .
\end{aligned}
$$

Case 2: $\mu=\infty$.

In this case, from the above argument, it is easy to see that the neck contains at least an infinite length geodesic.

In the end of this section, we give the proof of Theorem 1.2.

Proof of Theorem 1.2 By Theorem 3.1, we know that the images of the base map $\phi(M)$ and the bubbles $\omega^{j, k}\left(S^{2}\right), k=1, \ldots, K_{j}, j=1, \ldots, p$ are connected by geodesics on $N$. Next, we compute the sum of the lengths of these geodesics. By [28], the energy concentration at the punctures can be reduced to the study of $\left(\phi_{n}, \psi_{n}\right)$ on the long cylinders $P_{n}=\left[T_{n}^{1}, T_{n}^{2}\right] \times S^{1}$ with uniformly bounded energy $E\left(\phi_{n}, \psi_{n} ; P_{n}\right) \leq \Lambda$, where $1 \ll T_{n}^{1} \ll T_{n}^{2}$, i.e.

$$
\lim _{n \rightarrow \infty} \frac{1}{T_{n}^{1}}=0, \lim _{n \rightarrow \infty} \frac{T_{n}^{1}}{T_{n}^{2}}=0 .
$$

Moreover, there exists a constant $K>0$ independent of $n$ and $2 K$ sequences $\left\{a_{n}^{1}\right\},\left\{b_{n}^{1}\right\}$, $\left\{a_{n}^{2}\right\},\left\{b_{n}^{2}\right\}, \ldots,\left\{a_{n}^{K}\right\},\left\{b_{n}^{K}\right\}$, such that

$T_{n}^{1} \leq a_{n}^{1} \ll b_{n}^{1} \leq a_{n}^{2} \ll b_{n}^{2} \leq \cdots \leq a_{n}^{K} \ll b_{n}^{K} \leq T_{n}^{2}\left(a_{n}^{i} \ll b_{n}^{i}\right.$ means $\left.\lim _{n \rightarrow \infty} b_{n}^{i}-a_{n}^{i}=\infty\right)$

and $\left(b_{n}^{i}-a_{n}^{i}\right) \ll T_{n}^{2}$, i.e.

$$
\lim _{n \rightarrow \infty} \frac{b_{n}^{i}-a_{n}^{i}}{T_{n}^{2}}=0, \quad i=1, \ldots, K .
$$

Denote

$$
\begin{aligned}
& J_{n}^{j}:=\left[a_{n}^{j}, b_{n}^{j}\right] \times S^{1}, j=1, \ldots, K, \\
& I_{n}^{0}:=\left[T_{n}^{1}, a_{n}^{1}\right] \times S^{1}, I_{n}^{K}:=\left[b_{n}^{K}\right] \times S^{1}, I_{n}^{i}:=\left[b_{n}^{i}, a_{n}^{i+1}\right] \times S^{1}, i=1, \ldots, K-1 .
\end{aligned}
$$

Then

(1) $\forall i=0,1, \ldots, K$,

$$
\lim _{n \rightarrow \infty} \sup _{t \in I_{n}^{i}} \int_{[t, t+1] \times S^{1}}\left|\nabla \phi_{n}\right|^{2}=0 .
$$

The maps $\phi_{n}$ on $I_{n}^{i}$ are necks corresponding to collapsing homotopically nontrivial curves.

(2) $\forall j=0,1, \ldots, K$, there are finitely many Dirac-harmonic maps $\left(\sigma^{j, l} \xi^{j, l}\right): S^{2} \rightarrow N$, $l=1, \ldots, L_{j}$, such that 


$$
\begin{aligned}
& \lim _{n \rightarrow \infty} E\left(\phi_{n}, J_{n}^{j}\right)=\sum_{l=1}^{L_{j}} E\left(\sigma^{j, l}\right), \\
& \lim _{n \rightarrow \infty} E\left(\psi_{n}, J_{n}^{j}\right)=\sum_{l=1}^{L_{j}} E\left(\xi^{j, l}\right) .
\end{aligned}
$$

By Theorem 3.1, we know that map parts $\phi_{n}$ on $I_{n}^{i}$ are converging to the geodesics on $N$ of length

$$
\lim _{n \rightarrow \infty} \frac{1}{\sqrt{2 \pi}} \sqrt{\left|\operatorname{Re} \alpha\left(\phi_{n}, \psi_{n}\right)\right|}\left(a_{n}^{i+1}-b_{n}^{i}\right)
$$

and the spinor parts $\psi_{n}$ on $I_{n}^{i}$ are converging to 0 . By applying the neck analysis for Diracharmonic maps from a fixed domain in [19], the maps $\phi_{n}$ on $J_{n}^{j}$ are converging to a point, i.e., we get the no neck property. Thus, the maps $\phi_{n}$ on the whole cylinder $P_{n}$ converge to some geodesics on $N$. The sum of the lengths of these geodesics can be calculated as follows:

$$
\begin{aligned}
& \lim _{n \rightarrow \infty} \sum_{i=0}^{K-1} \frac{1}{\sqrt{2 \pi}} \sqrt{\left|\operatorname{Re} \alpha\left(\phi_{n}, \psi_{n}\right)\right|}\left(a_{n}^{i+1}-b_{n}^{i}\right) \\
& =\lim _{n \rightarrow \infty} \frac{1}{\sqrt{2 \pi}} \sqrt{\left|\operatorname{Re} \alpha\left(\phi_{n}, \psi_{n}\right)\right|}\left(T_{n}^{2}-T_{n}^{1}-\sum_{i=1}^{K}\left(b_{n}^{i}-a_{n}^{i}\right)\right) \\
& =\lim _{n \rightarrow \infty} \frac{1}{\sqrt{2 \pi}} \sqrt{\left|\operatorname{Re} \alpha\left(\phi_{n}, \psi_{n}\right)\right|}\left(T_{n}^{2}-T_{n}^{1}\right) \cdot \frac{T_{n}^{2}}{T_{n}^{2}-T_{n}^{1}}\left(1-\frac{T_{n}^{1}}{T_{n}^{2}}-\sum_{i=1}^{K} \frac{b_{n}^{i}-a_{n}^{i}}{T_{n}^{2}}\right) \\
& =\lim _{n \rightarrow \infty} \frac{1}{\sqrt{2 \pi}} \sqrt{\left|\operatorname{Re} \alpha\left(\phi_{n}, \psi_{n}\right)\right|}\left(T_{n}^{2}-T_{n}^{1}\right) \\
& =\lim _{n \rightarrow \infty} \frac{1}{\sqrt{2 \pi}} \sqrt{\left|\operatorname{Re} \alpha\left(\phi_{n}, \psi_{n}\right)\right|}\left|P_{n}\right|=\lim _{n \rightarrow \infty} \frac{1}{\sqrt{2 \pi}} \sqrt{\left|\operatorname{Re} \alpha\left(\phi_{n}, \psi_{n}\right)\right|} \frac{2 \pi^{2}}{l_{n}},
\end{aligned}
$$

which implies the conclusions of the theorem immediately.

\section{Proof of Theorem 1.4}

In this section, we shall calculate the second variation formula for the functional $L$ and define the notion of index of Dirac-harmonic maps. Similar calculation and definition for $\alpha$-Dirac-harmonic maps were given in [15]. Then, in analogy to [18], we shall give some geometric conditions on the target manifold which ensures that the energy identities hold for Dirac-harmonic maps from degenerating surfaces with bounded index.

Let $(\phi, \psi): M \rightarrow N$ be a Dirac-harmonic map. $\phi^{*}(T N)$ is the pull-back bundle over $M$. Let $V$ be a section of $L$. Consider the following variation of $(\phi, \psi)$ :

$$
\phi_{\tau}(x)=\exp _{\phi(x)}(\tau V), \psi_{\tau}(x)=\psi^{i}(x) \otimes \frac{\partial}{\partial y^{i}}\left(\phi_{\tau}(x)\right) .
$$

It is well-known that the following second variational formula for the energy of the map holds [22]

$$
\delta^{2} E(\phi)(V, V)=2 \int_{M}\left(\langle\nabla V, \nabla V\rangle-R(V, \nabla \phi, \nabla \phi, V)-\left\langle\tau(\phi), \nabla_{V} V\right\rangle\right) d t d \theta,
$$


where $\tau(\phi)=\Delta \phi-A(\phi)(d \phi, d \phi)$ is the tension field of the map $\phi$.

Next, we compute

$$
\left.\frac{d^{2}}{d \tau^{2}}\right|_{\tau=0} \int_{M}\left\langle\psi_{\tau}, \not D \psi_{\tau}\right\rangle=2 \int_{M}\left\langle\left.\frac{d}{d \tau}\right|_{\tau=0} \psi_{\tau},\left.\frac{d}{d \tau}\right|_{\tau=0} \not D \psi_{\tau}\right\rangle+\int_{M}\left\langle\psi_{\tau},\left.\frac{d^{2}}{d \tau^{2}}\right|_{\tau=0} \not D \psi_{\tau}\right\rangle .
$$

Choosing a local orthonormal basis $\left\{e_{\alpha}\right\}$ on $M$ such that $\left[e_{\alpha}, \frac{\partial}{\partial \tau}\right]=0, \nabla_{e_{\alpha}} e_{\beta}=0$ at a considered point, then we have

$$
\begin{aligned}
\frac{d}{d \tau} \not D \psi_{\tau} & =\frac{d}{d \tau}\left(e_{\alpha} \cdot \widetilde{\nabla}_{e_{\alpha}}\left(\psi^{i} \otimes \frac{\partial}{\partial y^{i}}\left(\phi_{\tau}\right)\right)\right) \\
& =\frac{d}{d \tau}\left(e_{\alpha} \cdot \nabla_{e_{\alpha}} \psi^{i} \otimes \frac{\partial}{\partial y^{i}}\left(\phi_{\tau}\right)+e_{\alpha} \cdot \psi^{i} \otimes \nabla_{e_{\alpha}} \frac{\partial}{\partial y^{i}}\left(\phi_{\tau}\right)\right) \\
& =e_{\alpha} \cdot \nabla_{e_{\alpha}} \psi^{i} \otimes \nabla_{\frac{\partial}{\partial \tau}} \frac{\partial}{\partial y^{i}}\left(\phi_{\tau}\right)+e_{\alpha} \cdot \psi^{i} \otimes \nabla_{\frac{\partial}{\partial \tau}} \nabla_{e_{\alpha}} \frac{\partial}{\partial y^{i}}\left(\phi_{\tau}\right) \\
& =e_{\alpha} \cdot \widetilde{\nabla}_{e_{\alpha}}\left(\psi^{i} \otimes \nabla_{\frac{\partial}{\partial \tau}} \frac{\partial}{\partial y^{i}}\left(\phi_{\tau}\right)\right)+e_{\alpha} \cdot \psi^{i} \otimes R\left(\frac{\partial}{\partial \tau}, e_{\alpha}\right) \frac{\partial}{\partial y^{i}}\left(\phi_{\tau}\right)
\end{aligned}
$$

and

$$
\begin{aligned}
\left.\frac{d^{2}}{d \tau^{2}}\right|_{\tau=0} \not D \psi_{\tau}= & e_{\alpha} \cdot \nabla_{e_{\alpha}} \psi^{i} \otimes \nabla_{\frac{\partial}{\partial \tau}} \nabla_{\frac{\partial}{\partial \tau}} \frac{\partial}{\partial y^{i}}\left(\phi_{\tau}\right)+e_{\alpha} \cdot \psi^{i} \otimes \nabla_{\frac{\partial}{\partial \tau}} \nabla_{e_{\alpha}} \nabla_{\frac{\partial}{\partial \tau}} \frac{\partial}{\partial y^{i}}\left(\phi_{\tau}\right) \\
& +e_{\alpha} \cdot \psi^{i} \otimes \nabla_{\frac{\partial}{\partial \tau}}\left(R\left(\frac{\partial}{\partial \tau}, e_{\alpha}\right) \frac{\partial}{\partial y^{i}}\left(\phi_{\tau}\right)\right) \\
= & e_{\alpha} \cdot \nabla_{e_{\alpha}} \psi^{i} \otimes \nabla_{\frac{\partial}{\partial \tau}} \nabla_{\frac{\partial}{\partial \tau}} \frac{\partial}{\partial y^{i}}\left(\phi_{\tau}\right)+e_{\alpha} \cdot \psi^{i} \otimes \nabla_{e_{\alpha}} \nabla_{\frac{\partial}{\partial \tau}} \nabla_{\frac{\partial}{\partial \tau}} \frac{\partial}{\partial y^{i}}\left(\phi_{\tau}\right) \\
& +e_{\alpha} \cdot \psi^{i} \otimes R\left(\frac{\partial}{\partial \tau}, e_{\alpha}\right) \nabla_{\frac{\partial}{\partial \tau}} \frac{\partial}{\partial y^{i}}\left(\phi_{\tau}\right) \\
& +e_{\alpha} \cdot \psi^{i} \otimes \nabla_{\frac{\partial}{\partial \tau}}\left(R\left(\frac{\partial}{\partial \tau}, e_{\alpha}\right) \frac{\partial}{\partial y^{i}}\left(\phi_{\tau}\right)\right) \\
= & e_{\alpha} \cdot \widetilde{\nabla}_{e_{\alpha}}\left(\psi^{i} \otimes \nabla_{\frac{\partial}{\partial \tau}} \nabla_{\frac{\partial}{\partial \tau}} \frac{\partial}{\partial y^{i}}\left(\phi_{\tau}\right)\right)+e_{\alpha} \cdot \psi^{i} \otimes R\left(\frac{\partial}{\partial \tau}, e_{\alpha}\right) \nabla_{\frac{\partial}{\partial \tau}} \frac{\partial}{\partial y^{i}}\left(\phi_{\tau}\right) \\
& +e_{\alpha} \cdot \psi^{i} \otimes \nabla_{\frac{\partial}{\partial \tau}}\left(R\left(\frac{\partial}{\partial \tau}, e_{\alpha}\right) \frac{\partial}{\partial y^{i}}\left(\phi_{\tau}\right)\right)
\end{aligned}
$$

Noting that

$$
R\left(\frac{\partial}{\partial \tau}, e_{\alpha}\right) \frac{\partial}{\partial y^{i}}\left(\phi_{\tau}\right)=R_{i k l}^{j} V^{k} d \phi^{l}\left(e_{\alpha}\right) \frac{\partial}{\partial y^{j}},
$$

we have

$$
\begin{aligned}
& \nabla_{\frac{\partial}{\partial \tau}}\left(R\left(\frac{\partial}{\partial \tau}, e_{\alpha}\right) \frac{\partial}{\partial y^{i}}\left(\phi_{\tau}\right)\right) \\
& \quad=R_{i k l ; p}^{j} V^{p} V^{k} d \phi^{l}\left(e_{\alpha}\right) \frac{\partial}{\partial y^{j}}+R\left(\nabla_{\frac{\partial}{\partial \tau}} \frac{\partial}{\partial \tau}, e_{\alpha}\right) \frac{\partial}{\partial y^{i}}\left(\phi_{\tau}\right)+R\left(\frac{\partial}{\partial \tau}, \nabla_{\frac{\partial}{\partial \tau}} e_{\alpha}\right) \frac{\partial}{\partial y^{i}}\left(\phi_{\tau}\right) .
\end{aligned}
$$


Combining these and using the equation $\not D \psi=0$, we get

$$
\begin{aligned}
& \left.\frac{d^{2}}{d \tau^{2}}\right|_{\tau=0} \int_{M}\left\langle\psi_{\tau}, \not D \psi_{\tau}\right\rangle \\
& =2 \int_{M}\left\langle\psi^{j} \otimes \nabla_{\frac{\partial}{\partial \tau}} \frac{\partial}{\partial y^{j}}\left(\phi_{\tau}\right), e_{\alpha} \cdot \widetilde{\nabla}_{e_{\alpha}}\left(\psi^{i} \otimes \nabla_{\frac{\partial}{\partial \tau}} \frac{\partial}{\partial y^{i}}\left(\phi_{\tau}\right)\right)\right. \\
& \left.+e_{\alpha} \cdot \psi^{i} \otimes R\left(\frac{\partial}{\partial \tau}, e_{\alpha}\right) \frac{\partial}{\partial y^{i}}\left(\phi_{\tau}\right)\right\rangle \\
& +\int_{M}\left\langle\psi, e_{\alpha} \cdot \widetilde{\nabla}_{e_{\alpha}}\left(\psi^{i} \otimes \nabla_{\frac{\partial}{\partial \tau}} \nabla_{\frac{\partial}{\partial \tau}} \frac{\partial}{\partial y^{i}}\left(\phi_{\tau}\right)\right)\right. \\
& +e_{\alpha} \cdot \psi^{i} \otimes R\left(\frac{\partial}{\partial \tau}, e_{\alpha}\right) \nabla_{\frac{\partial}{\partial \tau}} \frac{\partial}{\partial y^{i}}\left(\phi_{\tau}\right) \\
& +e_{\alpha} \cdot \psi^{i} \otimes\left(R_{i k l ; p}^{j} V^{p} V^{k} d \phi^{l}\left(e_{\alpha}\right) \frac{\partial}{\partial y^{j}}+R\left(\nabla_{\frac{\partial}{\partial \tau}} \frac{\partial}{\partial \tau}, e_{\alpha}\right) \frac{\partial}{\partial y^{i}}\left(\phi_{\tau}\right)\right. \\
& \left.\left.+R\left(\frac{\partial}{\partial \tau}, \nabla_{\frac{\partial}{\partial \tau}} e_{\alpha}\right) \frac{\partial}{\partial y^{i}}\left(\phi_{\tau}\right)\right)\right\rangle \\
& =2 \int_{M}\left\langle\psi^{j} \otimes \nabla_{\frac{\partial}{\partial \tau}} \frac{\partial}{\partial y^{j}}\left(\phi_{\tau}\right), e_{\alpha} \cdot \widetilde{\nabla}_{e_{\alpha}}\left(\psi^{i} \otimes \nabla_{\frac{\partial}{\partial \tau}} \frac{\partial}{\partial y^{i}}\left(\phi_{\tau}\right)\right)\right. \\
& \left.+e_{\alpha} \cdot \psi^{i} \otimes R\left(\frac{\partial}{\partial \tau}, e_{\alpha}\right) \frac{\partial}{\partial y^{i}}\left(\phi_{\tau}\right)\right\rangle \\
& +\int_{M}\left\langle\psi, e_{\alpha} \cdot \psi^{i} \otimes R\left(\nabla_{\frac{\partial}{\partial \tau}} \frac{\partial}{\partial \tau}, e_{\alpha}\right) \frac{\partial}{\partial y^{i}}\left(\phi_{\tau}\right)\right. \\
& +e_{\alpha} \cdot \psi^{i} \otimes\left(R_{i k l ; p}^{j} V^{p} V^{k} d \phi^{l}\left(e_{\alpha}\right) \frac{\partial}{\partial y^{j}}+R\left(\frac{\partial}{\partial \tau}, e_{\alpha}\right) \nabla_{\frac{\partial}{\partial \tau}} \frac{\partial}{\partial y^{i}}\left(\phi_{\tau}\right)\right. \\
& \left.\left.+R\left(\frac{\partial}{\partial \tau}, \nabla_{\frac{\partial}{\partial \tau}} e_{\alpha}\right) \frac{\partial}{\partial y^{i}}\left(\phi_{\tau}\right)\right)\right\rangle \text {. }
\end{aligned}
$$

By the fact that $\tau(\phi)=R(\phi, \psi)$ and

$$
\left\langle\psi, e_{\alpha} \cdot \psi^{i} \otimes R\left(\nabla_{\frac{\partial}{\partial \tau}} \frac{\partial}{\partial \tau}, e_{\alpha}\right) \frac{\partial}{\partial y^{i}}\left(\phi_{\tau}\right)\right\rangle=2\left\langle R(\phi, \psi), \nabla_{V} V\right\rangle,
$$

we obtain the following proposition:

Proposition 4.1 Let $(\phi, \psi): M \rightarrow N$ be a Dirac-harmonic map and $V$ be a smooth section of $\phi^{*} T N$. Then the second variational formula of the functional $L(\phi, \psi)$ with respect to the variations (4.1) is

$$
\begin{aligned}
\delta^{2} L(\phi, \psi)(V, V)= & \left.\frac{d^{2}}{d \tau^{2}}\right|_{\tau=0} L\left(\phi_{\tau}, \psi_{\tau}\right) \\
= & 2 \int_{M}(\langle\nabla V, \nabla V\rangle-R(V, \nabla \phi, \nabla \phi, V)) d t d \theta \\
& +2 \int_{M}\left\langle\psi^{j} \otimes \nabla_{V} \frac{\partial}{\partial y^{j}}, e_{\alpha} \cdot \widetilde{\nabla}_{e_{\alpha}}\left(\psi^{i} \otimes \nabla_{V} \frac{\partial}{\partial y^{i}}\right)\right. \\
& \left.+e_{\alpha} \cdot \psi^{i} \otimes R\left(V, e_{\alpha}\right) \frac{\partial}{\partial y^{i}}\right\rangle
\end{aligned}
$$




$$
\begin{aligned}
& +\int_{M}\left\langle\psi, e_{\alpha} \cdot \psi^{i} \otimes\left(R_{i k l ; p}^{j} V^{p} V^{k} d \phi^{l}\left(e_{\alpha}\right) \frac{\partial}{\partial y^{j}}\right.\right. \\
& \left.\left.+R\left(V, e_{\alpha}\right) \nabla_{V} \frac{\partial}{\partial y^{i}}+R\left(V, \nabla_{e_{\alpha}} V\right) \frac{\partial}{\partial y^{i}}\right)\right\rangle .
\end{aligned}
$$

Definition 4.2 Let $\Gamma\left(\phi^{*} T N\right)$ denote the linear space of smooth sections of $\phi^{*} T N$. The index of $(\phi, \psi)$ is defined as the maximal dimension of a linear subspace $\Xi$ of $\Gamma\left(\phi^{*} T N\right)$ on which the second variation of $L$ with respect to the variations (4.1) is negative, i.e., for any $V \in \Xi \subset \Gamma\left(\phi^{*} T N\right)$, there holds

$$
\delta^{2} L(\phi, \psi)(V, V)<0 .
$$

Before giving the proof of Theorem 1.4, we first prove the following theorem.

Theorem 4.3 Let $N$ be a compact Riemannian manifold. Let $\left(\phi_{n}, \psi_{n}\right):\left[-T_{n}, T_{n}\right] \times S^{1} \rightarrow N$ be a sequence of Dirac-harmonic maps with uniformly bounded energy

$$
E\left(\phi_{n}, \psi_{n} ;\left[-T_{n}, T_{n}\right] \times S^{1}\right) \leq \Lambda,
$$

where $\left[-T_{n}, T_{n}\right] \times S^{1}$ is a cylinder with standard flat metric $d s^{2}=d t^{2}+d \theta^{2}$ and $T_{n} \rightarrow \infty$ as $n \rightarrow \infty$. Suppose there is no energy concentration for $\left(\phi_{n}, \psi_{n}\right)$, if $\mu=\infty$ and Ric $c_{N} \geq$ $\lambda_{N}>0$, then the index of $\left(\phi_{n}, \psi_{n}\right)$ tends to infinity.

Since $\operatorname{Ric}_{N} \geq \lambda_{0}>0$, by the well-known Myers's theorem, we know that, if

$$
a \geq \frac{\pi}{\sqrt{\lambda_{0}(n-1)^{-1}}}+2 \epsilon,
$$

then there exists a tangent vector field $V_{0}(s)$ on $N$, which is smooth on $\gamma$, and is vanishing on $\left.\gamma\right|_{[0, \epsilon]}$ and $\left.\gamma\right|_{[a-\epsilon, a]}$, such that the second variation of length of $\gamma$ satisfies

$$
I_{\gamma}\left(V_{0}, V_{0}\right)=\int_{0}^{a}\left(\left\langle\nabla_{\dot{\gamma}} V_{0}, \nabla_{\dot{\gamma}} V_{0}\right\rangle-R\left(V_{0}, \dot{\gamma}, \dot{\gamma}, V_{0}\right)\right) d s<-\delta<0 .
$$

Recalling the curve

$$
\phi_{n}^{*}(t)=\frac{1}{2 \pi} \int_{0}^{2 \pi} \phi_{n}(t, \theta) d \theta
$$

let $s=s(t)$ be the arc-length parametrization of the curve $\phi_{n}^{*}(t)$ with $s(0)=0$. Set $s\left(t_{n}^{a}\right)=a$, by results in Sect. 3, we know that $\phi_{n}^{*}(s)$ converges to $\gamma$ on $[0, a]$. Moreover, according to Lemma 3.7, we have

$$
a=s\left(t_{n}^{a}\right)=\int_{0}^{t_{n}^{a}}\left|\phi_{n}^{*}\right|(t) d t=\left(\frac{1}{\sqrt{2 \pi}}+o(1)\right) \sqrt{\left|\operatorname{Re} \alpha\left(\phi_{n}, \psi_{n}\right)\right|} t_{n}^{a}
$$

which implies

$$
\lim _{n \rightarrow \infty} t_{n}^{a}=\infty \text { and } \sqrt{\left|\operatorname{Re} \alpha\left(\phi_{n}, \psi_{n}\right)\right|} t_{n}^{a} \leq C(a)
$$


Lemma 4.4 Under the assumptions of Theorem 4.3, for sufficiently large $n$, there exists a section $V_{n}$ of $\phi_{n}^{*}(T N)$, which is supported in $\left[0, t_{n}^{a}\right]$, such that

$$
\delta^{2} L\left(\phi_{n}, \psi_{n}\right)\left(V_{n}, V_{n}\right)<0 .
$$

Proof Let $P$ be projection from $T \mathbb{R}^{K}$ to $T N$. We define

$$
V_{n}(s, \theta)=P_{\phi_{n}(s, \theta)}\left(V_{0}(s)\right),
$$

where $s$ is the arc-length parametrization of $\phi_{n}^{*}(t)$ with $s(0)=0$. Then, it is easy to see that $V_{n}$ is a smooth section of $\phi_{n}^{*}(T N)$ which is supported in $\left[0, t_{k}^{a}\right] \times S^{1}$. Since $\phi_{n}(s, \theta) \rightarrow \gamma(s)$ in $C^{1}\left([0, a] \times S^{1}\right)$, we have

$$
V_{n}\left(\phi_{n}(s, \theta)\right) \rightarrow V_{0}(s) \text { in } C^{1}\left([0, a] \times S^{1}\right) .
$$

Next, we will show

$$
\lim _{n \rightarrow+\infty} \frac{1}{\sqrt{\left|\operatorname{Re} \alpha\left(\phi_{n}, \psi_{n}\right)\right|}} \delta^{2} L\left(\phi_{n}, \psi_{n}\right)\left(V_{n}, V_{n}\right)=2 \sqrt{2 \pi} I_{\gamma}\left(V_{0}, V_{0}\right) .
$$

By Proposition 4.1, we have

$$
\begin{aligned}
\delta^{2} L\left(\phi_{n}, \psi_{n}\right)\left(V_{n}, V_{n}\right) & \\
= & 2 \int_{\left(0, t_{n}^{a}\right) \times S^{1}}\left(\left\langle\nabla V_{n}, \nabla V_{n}\right\rangle-R\left(V_{n}, \nabla \phi_{n}, \nabla \phi_{n}, V_{n}\right)\right) d t d \theta \\
& +2 \int_{\left(0, t_{n}^{a}\right) \times S^{1}}\left\langle\psi^{j} \otimes \nabla_{V_{n}} \frac{\partial}{\partial y^{j}}, e_{\alpha} \cdot \widetilde{\nabla}_{e_{\alpha}}\left(\psi_{n}^{i} \otimes \nabla_{V_{n}} \frac{\partial}{\partial y^{i}}\right)\right. \\
& \left.+e_{\alpha} \cdot \psi_{n}^{i} \otimes R\left(V_{n}, e_{\alpha}\right) \frac{\partial}{\partial y^{i}}\right\rangle \\
& +\int_{\left(0, t_{n}^{a}\right) \times S^{1}}\left\langle\psi_{n}, e_{\alpha} \cdot \psi_{n}^{i} \otimes\left(R_{i k l ; p}^{j} V_{n}^{p} V_{n}^{k} d \phi_{n}^{l}\left(e_{\alpha}\right) \frac{\partial}{\partial y^{j}}\right.\right. \\
& \left.\left.+R\left(V_{n}, e_{\alpha}\right) \nabla_{V_{n}} \frac{\partial}{\partial y^{i}}+R\left(V_{n}, \nabla_{e_{\alpha}} V_{n}\right) \frac{\partial}{\partial y^{i}}\right)\right\rangle \\
:= & \mathbf{I}+\mathbf{I I}+\mathbf{I I I} .
\end{aligned}
$$

On one hand, using (4.3), it is easy to see that

$$
\begin{aligned}
\frac{\mathbf{I I}+\mathbf{I I I}}{\sqrt{\left|\operatorname{Re} \alpha\left(\phi_{n}, \psi_{n}\right)\right|}} & \leq \frac{C\left(N,\left\|V_{0}\right\|_{C^{1}}\right)}{\sqrt{\left|\operatorname{Re} \alpha\left(\phi_{n}, \psi_{n}\right)\right|}} \int_{\left(0, t_{n}^{a}\right) \times S^{1}}\left(\left|\psi_{n}\right|^{2}+\left|\psi_{n}\right|\left|\nabla \psi_{n}\right|+\left|\psi_{n}\right|^{2}\left|\nabla \phi_{n}\right|\right) \\
& \leq C\left(N,\left\|V_{0}\right\|_{C^{1}}\right)\left(\sqrt{\left|\operatorname{Re} \alpha\left(\phi_{n}, \psi_{n}\right)\right|}\right)^{3} t_{n}^{a} \rightarrow 0 .
\end{aligned}
$$

On the other hand, we get

$$
\begin{aligned}
\mathbf{I}= & 2 \int_{0}^{2 \pi} \int_{0}^{t_{n}^{a}}\left(\left\langle\nabla V_{n}, \nabla V_{n}\right\rangle-R\left(V_{n}, \nabla \phi_{n}, \nabla \phi_{n}, V_{n}\right)\right) d t d \theta \\
= & 2 \int_{0}^{2 \pi} \int_{0}^{t_{n}^{a}}\left(\left\langle\nabla_{\frac{\partial \phi_{n}}{\partial t}} V_{n}, \nabla_{\frac{\partial \phi_{n}}{\partial t}} V_{n}\right\rangle-R\left(V_{n}, \frac{\partial \phi_{n}}{\partial t}, \frac{\partial \phi_{n}}{\partial t}, V_{n}\right)\right) d t d \theta \\
& +2 \int_{0}^{2 \pi} \int_{0}^{t_{n}^{a}}\left(\left\langle\nabla_{\frac{\partial \phi_{n}}{\partial \theta}} V_{n}, \nabla_{\frac{\partial \phi_{n}}{\partial \theta}} V_{n}\right\rangle-R\left(V_{n}, \frac{\partial \phi_{n}}{\partial \theta}, \frac{\partial \phi_{n}}{\partial \theta}, V_{n}\right)\right) d t d \theta \\
= & \mathbf{I}_{1}+\mathbf{I}_{2} .
\end{aligned}
$$


Noting

$$
\begin{aligned}
\frac{\mathbf{I}_{1}}{\sqrt{\left|\operatorname{Re} \alpha\left(\phi_{n}, \psi_{n}\right)\right|}}= & 2 \int_{0}^{2 \pi} \int_{0}^{t_{n}^{a}}\left(\left\langle\nabla_{\frac{\partial \phi_{n}}{\partial s}} V_{n}, \nabla_{\frac{\partial \phi_{n}}{\partial s}} V_{n}\right\rangle-R\left(V_{n}, \frac{\partial \phi_{n}}{\partial s}, \frac{\partial \phi_{n}}{\partial s}, V_{n}\right)\right) \\
& \times \frac{\left|\frac{\partial s}{\partial t}\right|}{\sqrt{\left|\operatorname{Re} \alpha\left(\phi_{n}, \psi_{n}\right)\right|}} d t d \theta,
\end{aligned}
$$

we infer from Lemma 3.7

$$
\lim _{n \rightarrow+\infty} \frac{\mathbf{I}_{1}}{\sqrt{\left|\operatorname{Re} \alpha\left(\phi_{n}, \psi_{n}\right)\right|}}=2 \sqrt{2 \pi} I_{\gamma}\left(V_{0}, V_{0}\right)
$$

For the term $\mathbf{I}_{2}$, we have

$$
\begin{aligned}
& \frac{\mathbf{I}_{2}}{\sqrt{\left|\operatorname{Re} \alpha\left(\phi_{n}, \psi_{n}\right)\right|}} \\
& =\frac{2}{\sqrt{\left|\operatorname{Re} \alpha\left(\phi_{n}, \psi_{n}\right)\right|}} \int_{0}^{2 \pi} \int_{0}^{t_{n}^{a}}\left(\left\langle\nabla_{\frac{\partial \phi_{n}}{\partial \theta}} V_{n}, \nabla_{\frac{\partial \phi_{n}}{\partial \theta}} V_{n}\right\rangle-R\left(V_{n}, \frac{\partial \phi_{n}}{\partial \theta}, \frac{\partial \phi_{n}}{\partial \theta}, V_{n}\right)\right) d t d \theta \\
& \leq \frac{C}{\sqrt{\left|\operatorname{Re} \alpha\left(\phi_{n}, \psi_{n}\right)\right|}} \int_{0}^{2 \pi} \int_{0}^{t_{n}^{a}}\left|\frac{\partial \phi_{n}}{\partial \theta}\right|^{2} d t d \theta .
\end{aligned}
$$

For any given $T>0$, we set

$$
m_{n}=\left[\frac{t_{n}^{a}}{T}\right]+1,
$$

which tends to infinity as $n \rightarrow \infty$. By (4.3), there holds

$$
\sqrt{\left|\operatorname{Re} \alpha\left(\phi_{n}, \psi_{n}\right)\right|} m_{n} \leq C(T) .
$$

Hence, it follows that

$$
\begin{aligned}
\frac{\mathbf{I}_{2}}{\sqrt{\left|\operatorname{Re} \alpha\left(\phi_{n}, \psi_{n}\right)\right|}} & \leq \frac{C}{\sqrt{\left|\operatorname{Re} \alpha\left(\phi_{n}, \psi_{n}\right)\right|}} \int_{\cup_{i=0}^{m_{n}}[i T,(i+1) T] \times S^{1}}\left|\frac{\partial \phi_{n}}{\partial \theta}\right|^{2} d t d \theta \\
& \leq \frac{C m_{n} \sqrt{\left|\operatorname{Re} \alpha\left(\phi_{n}, \psi_{n}\right)\right|}}{\left|\operatorname{Re} \alpha\left(\phi_{n}, \psi_{n}\right)\right|} \frac{1}{m_{n}} \int_{\cup_{i=0}^{m_{n}}[i T,(i+1) T] \times S^{1}}\left|\frac{\partial \phi_{n}}{\partial \theta}\right|^{2} d t d \theta \\
& \leq \frac{C(T)}{m_{n}}\left(\frac{1}{\left|\operatorname{Re} \alpha\left(\phi_{n}, \psi_{n}\right)\right|} \int_{\cup_{i=0}^{m_{n}}[i T,(i+1) T] \times S^{1}}\left|\frac{\partial \phi_{n}}{\partial \theta}\right|^{2} d t d \theta\right) .
\end{aligned}
$$

In view of Lemma 3.2, we conclude that

$$
\lim _{n \rightarrow \infty} \frac{1}{\sqrt{\left|\operatorname{Re} \alpha\left(\phi_{n}, \psi_{n}\right)\right|}} \mathbf{I}_{2}=0 .
$$

Immediately, it follows that

$$
\lim _{n \rightarrow+\infty} \frac{1}{\sqrt{\left|\operatorname{Re} \alpha\left(\phi_{n}, \psi_{n}\right)\right|}} \delta^{2} L\left(\phi_{n}, \psi_{n}\right)\left(V_{n}, V_{n}\right)=2 \sqrt{2 \pi} I_{\gamma}\left(V_{0}, V_{0}\right) .
$$

Hence, for $n$ large enough, we have the desired inequality

$$
\delta^{2} L\left(\phi_{n}, \psi_{n}\right)\left(V_{n}, V_{n}\right)<0 .
$$

Thus, we complete the proof of this lemma. 
Proof of Theorem 4.3 Since $\mu=\infty$, then Theorem 3.1 tells us that the limit of the neck contains a geodesic of infinite length. For given $t_{0}$, by Lemma 2.7, we can always choose a suitable positive constant $t_{n}^{1}$ such that, when $n$ is large enough, the arc length $a$ of $\phi_{n}^{*}(t)$ on $\left[t_{0}, t_{0}+t_{n}^{1}\right]$ satisfies

$$
a>l_{N}=\frac{\pi}{\sqrt{\lambda_{0}(n-1)^{-1}}} .
$$

Therefore, by Lemma 4.4, there exists a section $V_{n}^{1}$ of $\phi_{n}^{*}(T N)$, which is 0 outside $\left[t_{0}, t_{0}+\right.$ $\left.t_{n}^{1}\right] \times S^{1}$, satisfying

$$
\delta^{2} L\left(\phi_{n}, \psi_{n}\right)\left(V_{n}^{1}, V_{n}^{1}\right)<0 .
$$

By the same method, for $t_{1}=t_{0}+t_{n}^{1}$, we can also pick $t_{n}^{2}>0$ and construct a section $V_{n}^{2}$, which is 0 outside $\left[t_{1}, t_{1}+t_{n}^{2}\right] \times S^{1}$, such that

$$
\delta^{2} L\left(\phi_{n}, \psi_{n}\right)\left(V_{n}^{2}, V_{n}^{2}\right)<0 .
$$

Since the limit of the neck contains a geodesic of infinite length, then, when $n$ is sufficiently large, there exist $i_{n}$ with $i_{n} \rightarrow \infty$ such that we can construct via the same way as above a series of sections $\left\{V_{n}^{3}, V_{n}^{4}, \ldots, V_{n}^{i_{n}}\right\}$ satisfying that for any $1 \leq i \leq i_{n}$ there holds

$$
\delta^{2} L\left(\phi_{n}, \psi_{n}\right)\left(V_{n}^{i}, V_{n}^{i}\right)<0 .
$$

Obviously, $V_{n}^{1}, V_{n}^{2}, \ldots, V_{n}^{i_{n}}$ are linearly independent. This means that the index of $\left(\phi_{n}, \psi_{n}\right)$ tends to infinity as $n \rightarrow \infty$. Thus, we complete the proof of the theorem.

Now, we prove Theorem 1.4.

Proof of Theorem 1.4 By Theorem 4.3, we know that the limit of $\phi_{n}$ on a neck domain consists of finite length geodesics. By [28], we can reduce the energy concentration at the nodes to the study of the asymptotic behaviour of a sequence of Dirac-harmonic maps from long cylinders $\left[-T_{n}, T_{n}\right] \times S^{1}$ as stated in Theorem 3.1. Moreover, according to Theorem 3.1, we have $\mu<\infty$ which implies $\lim _{n \rightarrow \infty}\left|\operatorname{Re} \alpha\left(\phi_{n}, \psi_{n}\right)\right| T_{n}=0$. Then energy identities follow immediately from Lemma 2.8 . The proof is finished.

Acknowledgments Open access funding provided by Max Planck Society.

Open Access This article is distributed under the terms of the Creative Commons Attribution 4.0 International License (http://creativecommons.org/licenses/by/4.0/), which permits unrestricted use, distribution, and reproduction in any medium, provided you give appropriate credit to the original author(s) and the source, provide a link to the Creative Commons license, and indicate if changes were made.

\section{References}

1. Balinsky, A., Evans, W.: Some recent results on Hardy-type inequalities. Appl. Math. Inf. Sci. 4(2), 191-208 (2010)

2. Chen, Q., Jost, J., Li, J., Wang, G.: Regularity theorems and energy identities for Dirac-harmonic maps. Math. Z. 251(1), 61-84 (2005)

3. Chen, Q., Jost, J., Li, J., Wang, G.: Dirac-harmonic maps. Math. Z. 254(2), 409-432 (2006)

4. Chen, Q., Jost, J., Sun, L., Zhu, M.: Estimates for solutions of Dirac equations and an application to a geometric elliptic-parabolic problem. J. Eur. Math. Soc. (JEMS) 21(3), 665-707 (2019)

5. Chen, Q., Jost, J., Sun, L., Zhu, M.: Dirac-geodesics and their heat flows. Calc. Var. Partial Differ. Equ. 54(3), 2615-2635 (2015) 
6. Chen, Q., Jost, J., Wang, G., Zhu, M.: The boundary value problem for Dirac-harmonic maps. J. Eur. Math. Soc. (JEMS) 15(3), 997-1031 (2013)

7. Chen, L., Li, Y., Wang, Y.: The refined analysis on the convergence behavior of harmonic map sequence from cylinders. J. Geom. Anal. 22(4), 942-963 (2012)

8. Chen, J., Tian, G.: Compactification of moduli sapce of harmonic mappings. Comment. Math. Helv. 74, 201-237 (1999)

9. Ding, W.: Lectures on heat flow of harmonic maps. Lecture notes at CTS, NTHU, Taiwan (1998)

10. Ding, W., Tian, G.: Energy identity for a class of approximate harmonic maps from surfaces. Commun. Anal. Geom. 3(3-4), 543-554 (1995)

11. Jost, J., Liu, L., Zhu, M.: Geometric analysis of the action functional of the nonlinear supersymmetric sigma model. MPI MIS Preprint: 77/2015

12. Jost, J., Liu, L., Zhu, M.: A global weak solution of the Dirac-harmonic map flow. Ann. Inst. H. Poincare Anal. Non Lineaire 34(7), 1851-1882 (2017)

13. Jost, J., Liu, L., Zhu, M.: Blow-up analysis for approximate Dirac-harmonic maps in dimension 2 with applications to the Dirac-harmonic heat flow. Calc. Var. Partial Differ. Equ. 56(4), 26 (2017)

14. Jost, J., Liu, L., Zhu, M.: Energy identity for a class of approximate Dirac-harmonic maps from surfaces with boundary. Ann. Inst. H. Poincare Anal. Non Lineaire 36(2), 365-387 (2019)

15. Jost, J., Liu, L., Zhu, M.: Geometric analysis of a mixed elliptic-parabolic conformally invariant boundary value problem. MPI MIS Preprint 41/2018

16. Jost, J., Zhou, C., Zhu, M.: Vanishing Pohozaev constant and removability of singularities. J. Differ. Geom. 111(1), 91-144 (2019)

17. Lawson, H., Michelsohn, M.: Spin Geometry, vol. 38. Princeton University Press, Princeton (1989)

18. Li, Y., Liu, L., Wang, Y.: Blow-up behavior of harmonic maps with finite index. Calc. Var. Partial Differ. Equ. 56(5), 16 (2017)

19. Liu, L.: No neck for Dirac-harmonic maps. Calc. Var. Partial Differ. Equ. 52(1-2), 1-15 (2015)

20. Parker, T.H.: Bubble tree convergence for harmonic maps. J. Differ. Geom. 44, 595-633 (1996)

21. Qing, J., Tian, G.: Bubbling of the heat flows for harmonic maps from surfaces. Commun. Pure Appl. Math. 50(4), 295-310 (1997)

22. Sacks, J., Uhlenbeck, K.: The existence of minimal immersions of 2-spheres. Ann. Math. 113, 1-24 (1981)

23. Sharp, B.: Compactness of minimal hypersurfaces with bounded index. J. Differ. Geom. 106(2), 317-339 (2017)

24. Sharp, B., Zhu, M.: Regularity at the free boundary for Dirac-harmonic maps from surfaces. Calc. Var. Partial Differ. Equ. 55(2), 55:27 (2016)

25. Simon, L.: Asymptotics for a class of nolinear evolution equations with applications to geometric problems. Ann. Math. 118, 525-571 (1983)

26. Zhao, L.: Energy identities for Dirac-harmonic maps. Calc. Var. Partial Differ. Equ. 28(1), 121-138 (2007)

27. Zhu, M.: Regularity of weakly Dirac-harmonic maps to hypersurfaces. Ann. Glob. Anal. Geom. 35(4), 405-412 (2009)

28. Zhu, M.: Dirac-harmonic maps from degenerating spin surfaces. I. The Neveu-Schwarz case. Calc. Var. Partial Differ. Equ. 35(2), 169-189 (2009)

29. Zhu, M.: Harmonic maps from degenerating Riemann surfaces. Math. Z. 264(1), 63-85 (2010)

Publisher's Note Springer Nature remains neutral with regard to jurisdictional claims in published maps and institutional affiliations. 